\title{
The ALMA Fornax Cluster Survey I: stirring and stripping of the molecular gas in cluster galaxies
}

\author{
Nikki Zabel, ${ }^{1 \star}$ Timothy A. Davis ${ }^{\oplus},{ }^{1}$ Matthew W. L. Smith ${ }^{\oplus},{ }^{1}$ Natasha Maddox ${ }^{\odot}, 2,3$ \\ George J. Bendo ${ }^{\odot}, 4$ Reynier Peletier ${ }^{\odot}, 5$ Enrichetta Iodice, ${ }^{6}$ Aku Venhola, ${ }^{7}$ \\ Maarten Baes ${ }^{\oplus},{ }^{8}$ Jonathan I. Davies, ${ }^{1}$ Ilse de Looze ${ }^{\bullet},{ }^{9,10,11}$ Haley Gomez, ${ }^{1}$ \\ Marco Grossi ${ }^{\oplus}, 12$ Jeffrey D. P. Kenney, ${ }^{13}$ Paolo Serra, ${ }^{14}$ Freeke van de Voort ${ }^{\circledR}, 15,16$ \\ Catherine Vlahakis ${ }^{17}$ and Lisa M. Young ${ }^{18,19}$
}

Affiliations are listed at the end of the paper

Accepted 2018 November 26. Received 2018 November 20; in original form 2018 October 11

\begin{abstract}
We present the first results of the ALMA Fornax Cluster Survey: a complete ALMA survey of all members of the Fornax galaxy cluster that were detected in $\mathrm{HI}$ or in the far-infrared with Herschel. The sample consists of a wide variety of galaxy types, ranging from giant ellipticals to spiral galaxies and dwarfs, located in all (projected) areas of the cluster. It spans a mass range of $10^{\sim .5-11} \mathrm{M}_{\odot}$. The $\mathrm{CO}(1-0)$ line was targeted as a tracer for the cold molecular gas, along with the associated $3 \mathrm{~mm}$ continuum. $\mathrm{CO}$ was detected in 15 of the 30 galaxies observed. All 8 detected galaxies with stellar masses below $3 \times 10^{9} \mathrm{M}_{\odot}$ have disturbed molecular gas reservoirs; only 7 galaxies are regular/undisturbed. This implies that Fornax is still a very active environment, having a significant impact on its members. Both detections and non-detections occur at all projected locations in the cluster. Based on visual inspection, and the detection of molecular gas tails in alignment with the direction of the cluster centre, in some cases ram pressure stripping is a possible candidate for disturbing the molecular gas morphologies and kinematics. Derived gas fractions in almost all galaxies are lower than expected for field objects with the same mass, especially for the galaxies with disturbed molecular gas, with differences of sometimes more than an order of magnitude. The detection of these disturbed molecular gas reservoirs reveals the importance of the cluster environment for even the tightly bound molecular gas phase.
\end{abstract}

Key words: galaxies: clusters: general - galaxies: clusters: individual: Fornax - galaxies: evolution-galaxies: ISM.

\section{INTRODUCTION}

It has long been known that galaxies in cluster environments evolve differently from their counterparts in the field. In particular, the relative number of early-type galaxies in cluster environments is significantly higher than in the field (e.g. Oemler 1974; Dressler 1980). In addition, the galaxies that are present in clusters have a smaller atomic gas reservoir than their counterparts in the field (Haynes, Giovanelli \& Chincarini 1984; Cayatte et al. 1990; Solanes et al. 2001; Gavazzi et al. 2005). The clustering of galaxies generates an extreme environment, which is likely capable of quenching the star formation in galaxies, transforming them from blue, late-type galaxies to red ellipticals.
Over the years, various processes have been proposed as the responsible mechanism for this transformation. Ram pressure stripping (RPS) was first suggested as a candidate by Gunn \& Gott (1972), and similarly viscous stripping by Nulsen (1982), starvation by Larson, Tinsley \& Caldwell (1980), and thermal evaporation by Cowie \& Songaila (1977). Furthermore, there are galaxy-galaxy interactions, such as harassment (Moore et al. 1996) and mergers. So called pre-processing, which takes place at higher redshifts when the clusters are first formed and the galaxies' velocities are still relatively low, also plays a role in shaping the galaxies, in the form of minor mergers and tidal interactions (Fujita 2004; Mihos 2004; see Boselli \& Gavazzi 2006 for an extended review). The relative importance of the different mechanisms is still poorly understood. In any case, it is clear that the cluster environment plays a fundamental role in galaxy evolution, especially keeping in mind that 
$\sim 40$ per cent of galaxies live in groups or clusters (e.g. Robotham et al. 2011), and the majority of the local galaxies live in groups (e.g. Zabludoff \& Mulchaey 1998).

It is well known that the atomic gas in galaxies is affected by the above-mentioned processes. The situation is more complicated for the molecular gas, because it is more tightly bound to the galaxy and distributed more centrally. The debate about this has therefore been more lively. Early research often concluded that the molecular gas in cluster galaxies is the same as that in field galaxies, and is unaffected by the cluster environment (e.g. Stark et al. 1986; Kenney \& Young 1989; Casoli et al. 1991; Boselli, Casoli \& Lequeux 1995; Boselli \& Gavazzi 2006). It was not until more recently that indications of deficiency, that is a lower mass than expected based on statistics of similar galaxies in the field, were observed for the molecular gas as well (e.g. Vollmer et al. 2008; Fumagalli et al. 2009; Boselli et al. 2014) and also for dust (e.g. Cortese et al. 2010, 2012), although these deficiencies are smaller than for H I. On average galaxies that are $\mathrm{HI}$ deficient by a factor of $\sim 10$ are $\mathrm{CO}$ deficient by a factor of $\sim 2$. Lee et al. (2017) report examples of three galaxies in the Virgo cluster that are ram pressure stripped of their molecular gas as well as their atomic gas. At higher redshifts, evidence of molecular gas stripping and deficiencies in clusters has also recently been observed (e.g. Stach et al. 2017; Noble et al. 2018; Wang et al. 2018), although cluster galaxies with molecular gas contents similar to (e.g. Rudnick et al. 2017) or even higher than (e.g. Hayashi et al. 2018) field galaxies are found as well.

Because molecular gas is the direct fuel for star formation, the effects of the cluster environment on this phase of the interstellar medium (ISM) have immediate consequences for the star formation rate (SFR) of the host galaxy. If it is directly affected by environmental processes, this could have important implications for the quenching of cluster members and therefore for galaxy evolution as a whole.

The goal of this work is to investigate whether the cluster environment indeed affects the molecular gas in galaxies, and if so, attempt to identify which processes are mainly responsible for this. In order to do this, we focus our attention on the Fornax cluster. Fornax is among the two nearest galaxy clusters, together with the Virgo cluster. They are located at 19.95 (Tonry et al. 2001) and $16.8 \mathrm{Mpc}$ (NASA/IPAC Extragalactic Database), respectively. Both clusters are therefore ideal laboratories to study the effects of the cluster environment on galaxies at high resolution. Extensive catalogues exist for both clusters, compiled by Binggeli, Sandage \& Tammann (1985) for Virgo and by Ferguson (1989) for Fornax. Other, more recent studies of the Virgo cluster include the deep optical Next Generation Virgo Survey (NGVS; Ferrarese et al. 2012), the Herschel Virgo Cluster Survey (HeViCS; Davies et al. 2010) in the far-infrared (FIR), the GALEX Ultraviolet Virgo Cluster Survey (GUViCS; Boselli et al. 2011) in the ultraviolet, and the blind narrow-band $\mathrm{H} \alpha+[\mathrm{N}$ II] imaging survey Virgo Environmental Survey Tracing Ionised Gas Emission (VESTIGE; Boselli et al. 2018). Located in the Southern hemisphere, Fornax has been studied less than its northern counterpart. However, recently more and more studies of the Fornax cluster have appeared. These include the optical Fornax Deep Survey (FDS; Iodice et al. 2016, 2017; Venhola et al. 2017, 2018; Peletier et al. in prep.), the Herschel Fornax Cluster Survey (HeFoCS; Davies et al. 2013), the integral-field spectroscopic survey Fornax3D (Sarzi et al. 2018), the blind $\mathrm{HI}$ Australia Telescope Compact Array (ATCA) survey (Lee-Waddell et al. 2018), and soon the MeerKAT Fornax H I and radio continuum survey (Serra et al. 2016).
There are some fundamental differences between both clusters that add to the importance of studying the Fornax cluster in addition to the Virgo cluster. First, Fornax is much smaller than Virgo, with Virgo being $~ 10$ times as massive as Fornax (Jordán et al. 2007). It is home to 2000 galaxies, while Fornax harbours only 350 (detected at the time of the catalogues mentioned above, both complete in magnitudes up to $B_{T} \approx 18$ and containing members with magnitudes up to $B_{T} \approx 20$ ). Despite its smaller size, the Fornax cluster has a number density of roughly 3 times that of Virgo. Fornax is also more regular and dynamically evolved than Virgo, and has a lower velocity dispersion. Because it is more relaxed, environment- and density-related effects are easier to identify in Fornax: Galaxies in its centre will be more strongly affected by density effects than galaxies in the outskirts. In Virgo these effects are harder to identify, because it is still in the process of assembling. The central hot gas density in Fornax is 4 times lower than that in Virgo, and its temperature is twice as low (Schindler, Binggeli \& Böhringer 1999; Paolillo et al. 2002; Scharf, Zurek \& Bureau 2005). These differences suggest that ram pressure stripping plays less of a role in the Fornax cluster, compared to Virgo. According to Davies et al. (2013) ram pressure stripping should be a factor 16 less important in Fornax, based on the equation from Gunn \& Gott (1972): $P_{\mathrm{r}} \approx \rho_{e} v^{2}$, where $P_{\mathrm{r}}$ is the ram pressure, $\rho_{e}$ is the intracluster density, and $v$ is the velocity of the galaxy. The higher number density in Fornax, on the other hand, suggests that galaxy-galaxy interactions are more important. In this work we turn to a resolved study of the ISM in Fornax galaxies to investigate these processes further.

Horellou, Casoli \& Dupraz (1995) carried out an H I and ${ }^{12} \mathrm{CO}(1-$ $0)$ survey of 21 spirals and lenticulars in the Fornax cluster, using the Nançay radio telescope (France) and the Swedish-ESO Submillimetre Telescope (SEST; Booth et al. 1989), respectively. They detected 16 galaxies in $\mathrm{HI}$, and 11 were detected in $\mathrm{CO}$. They found that on average the $\mathrm{CO}$ emission of Fornax galaxies is weak: about 5 times lower than that of spirals in the Virgo cluster. From this it follows that the corresponding molecular gas masses are low as well: They found $\mathrm{H}_{2}$ masses that are about 10 times lower than the atomic gas masses. They attribute the decreased molecular gas masses to reduced star formation activity, and argue that it is in agreement with low far-infrared, radio continuum, and $\mathrm{H} \alpha$ luminosities. They comment, however, that although the $\mathrm{CO}$ emission found for the Fornax galaxies is low compared to that in infrared-selected samples, that may be typical for spirals in optically selected samples. In this work we revisit the $\mathrm{CO}(J=1-0)$ in the Fornax cluster and investigate whether these observations can be confirmed.

The ALMA Fornax Cluster Survey is a complete survey of the 30 Fornax cluster members that were detected in three or more Herschel Space Observatory (Pilbratt et al. 2010) bands with the Herschel Fornax Cluster Survey (Fuller et al. 2014) or in H I (Waugh et al. 2002; Loni et al. in prep.; based on ATCA data). The CO(10) rotational line (rest frequency: $115.271 \mathrm{GHz}$ ) was observed to create spatially resolved maps of the cold molecular gas and its kinematics in these galaxies. The survey covers a range of different galaxy stellar masses and morphologies.

A full description of the sample can be found in Section 2. The observations, data reduction, and ancillary data are described in Section 3. In Section 4 we present moment maps of the $\mathrm{CO}$ emission of the detected galaxies, as well as their position-velocity diagrams (PVDs) and spectra, and a comparison with optical observations. $\mathrm{H}_{2}$ masses are estimated and compared with the expected $\mathrm{H}_{2}$ masses for field galaxies. In Section 5 we discuss the results, and the morphologies and kinematics of the galaxies in the sample. Various 
environmental processes are considered as possible candidates for the irregularities observed, and the surprising detection of several dwarf galaxies is discussed. In Section 6 we summarise the work, and distil conclusions. Although accurate distance measurements are available for some of the AlFoCS galaxies, here we adopt the distance to the Fornax cluster (19.95 Mpc; Tonry et al. 2001) as a common distance to all galaxies.

\section{SAMPLE SELECTION}

Our sample is based on the Fornax Cluster Catalogue (FCC; Ferguson 1989). From this catalogue, galaxies with stellar masses $>3 \times 10^{8} \mathrm{M}_{\odot}$ were selected to ensure high enough metallicity to detect $\mathrm{CO}$. Furthermore, galaxies were selected to contain dust (Fuller et al. 2014) or H I down to $\sim 3 \times 10^{7} \mathrm{M}_{\odot}$ (Waugh et al. 2002; Loni et al. in prep.; based on ATCA data). This suggests ongoing star formation activity, and therefore the presence of a molecular gas reservoir. Whether a galaxy was selected based on its FIR emission or HI content is listed in Table 1. The application of these criteria on the FCC leads to a sample of 30 galaxies, spanning a wide range of morphological types, varying from giant ellipticals to irregular dwarfs. A wide range of locations within the cluster is covered by the survey. This is shown in Fig. 1, where we compare the locations of the AlFoCS galaxies with the locations of the galaxies in the FDS (Iodice et al. 2016, 2017; Venhola et al. 2017, 2018; Peletier et al., in prep.). The FDS is a recent optical survey of the Fornax cluster, containing 573 galaxies, and is described in more detail in Section 3.3. The FDS galaxies are shown as black dots, and the galaxies targeted here are shown as red stars. The brightest cluster galaxy (BCG) NGC1399 is shown as a bigger yellow star, and the dotted line represents the virial radius of the cluster according to Drinkwater et al. (2001). The central galaxy of the currently infalling subgroup in the south-east of the figure, NGC1316, is indicated with a cyan star. Aside from a slight $(<10$ percent $)$ deficiency of galaxies in the innermost ( $\sim 350 \mathrm{kpc}$ or 1 degree) radius of the cluster centre (defined as the location of NGC1399), the AlFoCS targets are spread evenly among the cluster galaxies: They are located in all directions from the cluster centre, and both close to the central galaxy and outside the virial radius. There are no observations in the infalling subgroup around NGC1316, as this area was not covered by Herschel.

To confirm that all the targets are indeed cluster members, we create a caustic diagram of all galaxies with known velocities: the (projected) velocities of the galaxies (corrected for the velocity of the cluster and galaxy-to-galaxy velocity dispersion within the cluster) of the cluster as a function of their distance from the cluster centre. This is shown in Fig. 2. The mean velocity and velocity dispersion of the Fornax cluster were taken from Drinkwater et al. (2001), and are equal $1493 \mathrm{~km} \mathrm{~s}^{-1}$ and $374 \mathrm{~km} \mathrm{~s}^{-1}$, respectively. The velocities of the individual galaxies are a combination of velocities from the FCC, the 2dF Galaxy Redshift Survey (Colless et al. 2001; Drinkwater et al. 1999), and the 2MASS Redshift Survey (Huchra et al. 2012). Note that velocity information is unavailable for 470 of the 573 FDS galaxies, and these were omitted from the figure. The solid lines represent the escape velocities at each projected distance from the cluster centre, assuming a Navarro-Frenk-White density profile for the cluster dark matter distribution (Navarro, Frenk \& White 1997). They were derived using equations 7 and 16 from Shull (2014), featuring a dark matter concentration parameter, which was estimated using equation 3 from Coe (2010). The dotted line again represents the virial radius, and the colours are the same as in Fig. 1. All AlFoCS galaxies shown here have velocities well below the escape velocity at their location, and are distributed evenly in the caustic space.

The locations, velocities, and stellar masses of the galaxies observed are listed in Table 1.

\section{OBSERVATIONS AND DATA REDUCTION}

\subsection{ALMA data}

Atacama Large Millimeter/submillimeter Array (ALMA) observations of the ${ }^{12} \mathrm{CO}(1-0)$ line in 29 AlFoCS targets were carried out under project 2015.1.00497.S (PI: Timothy Davis). ALMA's $12 \mathrm{~m}$ configuration was used, which has a primary beam size of $\sim 55 \mathrm{arcsec}$ at $\sim 115 \mathrm{GHz}$. In cases where the FIR emission of the galaxy extends beyond this scale, multiple pointings are combined into a mosaic to ensure that $\mathrm{CO}$ is observed all the way to the outskirts of the galaxy. The largest recoverable scale is 25 arcsec. Band 3 observations were performed between 2016 January 7 and 12, subdivided in three Scheduling Blocks (SBs) in order to meet the sensitivity requirements of the different targets whilst keeping maximum efficiency: single fields, small mosaics, and dwarfs. The first SB consists of one Execution Block (EB): uid _-_A002_Xaeaf96_X515. The small mosaics are divided over two Execution Blocks: uid___A002_Xaec9ef_X5c0 and uid___A002_Xaec9ef_X88a. The same is true for the dwarfs, which are divided over Execution Blocks uid___A002_Xaecf7b_X32d4 and uid_-_A002_Xaecf7b_X3943. For each SB one spectral window was centred at $114.756,114.547$, and $114.716 \mathrm{GHz}$, respectively, to target the ${ }^{12} \mathrm{CO}(1-0)$ rotational line. The bandwidths are $1.875 \mathrm{GHz}$, covering 3840 channels. The other spectral windows, covering 128 channels each with total bandwidths of $2 \mathrm{GHz}$, were used to target the band 3 continuum of the individual galaxies. Their central frequencies, along with other details of the observations, are listed in Table 2. The expected calibration uncertainty of the data is 10 percent. Synthesized beam sizes and the sensitivities achieved are listed in Table 3.

\subsubsection{Data reduction}

The data were calibrated manually using the Common Astronomy Software Applications package (CASA, version 5.1.1; McMullin et al. 2007), using standard ALMA calibration scripts. ${ }^{1}$ Several antennas were flagged manually, mostly because of high system temperatures or outliers in the data of the flux calibrator. The resulting 'dirty' images were then 'cleaned' using the tCLEAN algorithm (Högbom 1974) in CASA. In cases where both CO and continuum are detected, a continuum estimate is created using the full line-free bandwidth and subtracted from the channels containing the $\mathrm{CO}$ line using the uvcont sub command. Cleaning of the channels containing the $\mathrm{CO}$ line was done interactively, using a natural weighting scheme [equivalent to a Briggs weighting scheme (Briggs 1995) with a robust parameter of 2]. Many of the sources have extended emission, and using natural weighting will help ensure that this is recovered in the data. This choice also maximizes the signal to noise at the cost of decreased spatial resolution. The channel widths of most final data cubes are $10 \mathrm{~km} \mathrm{~s}^{-1}$, as is usually chosen for this type of data (e.g. Alatalo et al. 2013), and the pixel sizes are 0.5 arcsec.

\footnotetext{
${ }^{1}$ The scripts used can be found on https://github.com/NikkiZabel/AlFoCS _data_reduction_scripts
} 
Table 1. Key properties of the galaxies in the sample.

\begin{tabular}{|c|c|c|c|c|c|c|}
\hline $\begin{array}{l}\text { Common name } \\
- \\
\text { (1) }\end{array}$ & $\begin{array}{l}\text { FCC \# } \\
- \\
(2)\end{array}$ & $\begin{array}{l}\text { RA } \\
(\mathrm{J} 2000) \\
(3)\end{array}$ & $\begin{array}{l}\text { Dec. } \\
\text { (J2000) } \\
(4)\end{array}$ & $\begin{array}{l}c z \\
\left(\mathrm{~km} \mathrm{~s}^{-1}\right) \\
(5)\end{array}$ & $\begin{array}{l}M_{\star} \\
\left(\log \left(M_{\odot}\right)\right) \\
(6)\end{array}$ & $\begin{array}{l}\text { Selection } \\
- \\
(7)\end{array}$ \\
\hline FCC32 & 32 & $03^{\mathrm{h}} 24^{\mathrm{m}} 52^{\mathrm{s}} .4$ & $-35^{\circ} 26^{\prime} 08^{\prime \prime}$ & $1319^{\diamond}$ & $9.23_{-0.07}^{+0.04} *$ & FIR \\
\hline FCC44 & 44 & $03^{\mathrm{h}} 26^{\mathrm{m}} 07^{\S} .4$ & $-35^{\circ} 07^{\prime} 39^{\prime \prime}$ & $1233^{\diamond}$ & $8.50_{-0.17}^{+0.07} *$ & FIR \\
\hline NGC1351A & 67 & $03^{\mathrm{h}} 28^{\mathrm{m}} 48^{\mathrm{s}} .7$ & $-35^{\circ} 10^{\prime} 41^{\prime \prime}$ & 1354 & $9.45 \dagger$ & FIR, H I \\
\hline MGC-06-08-024 & 90 & $03^{\mathrm{h}} 31^{\mathrm{m}} 08^{\mathrm{s}} \cdot 2$ & $-36^{\circ} 17^{\prime} 25^{\prime \prime}$ & $1814^{\diamond}$ & $8.98 \dagger$ & FIR, H I \\
\hline FCC102 & 102 & $03^{\mathrm{h}} 32^{\mathrm{m}} 10^{\mathrm{s}} .7$ & $-36^{\circ} 13^{\prime} 15^{\prime \prime}$ & $1722 \ddagger$ & $8.36_{-0.10}^{+0.08} *$ & $\mathrm{HI}$ \\
\hline ESO358-G015 & 113 & $03^{\mathrm{h}} 33^{\mathrm{m}} 06^{\mathrm{s}} 8$ & $-34^{\circ} 48^{\prime} 29^{\prime \prime}$ & 1389 & $8.88 \dagger$ & FIR, H I \\
\hline ESO358-16 & 115 & $03^{\mathrm{h}} 33^{\mathrm{m}} 09^{\mathrm{s}} \cdot 2$ & $-35^{\circ} 43^{\prime} 07^{\prime \prime}$ & $1701^{\diamond}$ & $8.32_{-0.09}^{+0.07} *$ & $\mathrm{HI}$ \\
\hline FCC117 & 117 & $03^{\mathrm{h}} 33^{\mathrm{m}} 14^{\mathrm{s}} .6$ & $-37^{\circ} 49^{\prime} 11^{\prime \prime}$ & - & $7.77_{-0.20}^{+0.18} *$ & FIR \\
\hline FCC120 & 120 & $03^{\mathrm{h}} 33^{\mathrm{m}} 34^{\mathrm{s}} .2$ & $-36^{\circ} 36^{\prime} 21^{\prime \prime}$ & $849 \ddagger$ & $8.50_{-0.09}^{+0.07} *$ & $\mathrm{HI}$ \\
\hline NGC1365 & 121 & $03^{\mathrm{h}} 33^{\mathrm{m}} 36^{\mathrm{s}} .4$ & $-36^{\circ} 08^{\prime} 25^{\prime \prime}$ & $1638^{\diamond}$ & $11.16 \dagger$ & FIR, H I \\
\hline NGC1380 & 167 & $03^{\mathrm{h}} 36^{\mathrm{m}} 27^{\mathrm{s}} \cdot 6$ & $-34^{\circ} 58^{\prime} 34^{\prime \prime}$ & $1878^{\diamond}$ & $10.98 \dagger$ & FIR \\
\hline FCC177 & 177 & $03^{\mathrm{h}} 36^{\mathrm{m}} 47^{\mathrm{s}} .5$ & $-34^{\circ} 44^{\prime} 23^{\prime \prime}$ & $1562^{\diamond}$ & $10.4_{-0.02}^{+0.01} *$ & FIR \\
\hline NGC1386 & 179 & $03^{\mathrm{h}} 36^{\mathrm{m}} 46^{\mathrm{s}} .2$ & $-35^{\circ} 59^{\prime} 58^{\prime \prime}$ & $869^{\diamond}$ & $10.5 \dagger$ & FIR \\
\hline NGC1387 & 184 & $03^{\mathrm{h}} 36^{\mathrm{m}} 57^{\mathrm{s}} .0$ & $-35^{\circ} 30^{\prime} 24^{\prime \prime}$ & $1303^{\diamond}$ & $10.77 \dagger$ & FIR \\
\hline FCC198 & 198 & $03^{\mathrm{h}} 37^{\mathrm{m}} 42^{\mathrm{s}} \cdot 7$ & $-37^{\circ} 12^{\prime} 30^{\prime \prime}$ & - & $8.09_{-0.07}^{+0.05} *$ & FIR \\
\hline FCC206 & 206 & $03^{\mathrm{h}} 38^{\mathrm{m}} 13^{\mathrm{s}} .5$ & $-37^{\circ} 17^{\prime} 25^{\prime \prime}$ & $1403^{\diamond}$ & $9.01_{-0.10}^{+0.07} *$ & FIR \\
\hline FCC207 & 207 & $03^{\mathrm{h}} 38^{\mathrm{m}} 19^{\mathrm{s}} \cdot 3$ & $-35^{\circ} 07^{\prime} 45^{\prime \prime}$ & $1421^{\diamond}$ & $8.78_{-0.05}^{+0.04} *$ & FIR \\
\hline NGC1427A & 235 & $03^{\mathrm{h}} 40^{\mathrm{m}} 09^{\mathrm{s}} \cdot 3$ & $-35^{\circ} 37^{\prime} 28^{\prime \prime}$ & $2029^{\diamond}$ & $9.78 \dagger$ & FIR, H I \\
\hline FCC261 & 261 & $03^{\mathrm{h}} 41^{\mathrm{m}} 21^{\mathrm{s}} .5$ & $-33^{\circ} 46^{\prime} 09^{\prime \prime}$ & $1710 \ddagger$ & $8.58 \dagger$ & FIR \\
\hline PGC013571 & 263 & $03^{\mathrm{h}} 41^{\mathrm{m}} 32^{\mathrm{s}} \cdot 6$ & $-34^{\circ} 53^{\prime} 18^{\prime \prime}$ & $1725^{\diamond}$ & $9.2 \dagger$ & FIR, H I \\
\hline FCC 282 & 282 & $03^{\mathrm{h}} 42^{\mathrm{m}} 45^{\mathrm{s}} \cdot 3$ & $-33^{\circ} 55^{\prime} 14^{\prime \prime}$ & $1266 \ddagger$ & $9.0 \dagger$ & FIR \\
\hline NGC1437A & 285 & $03^{\mathrm{h}} 43^{\mathrm{m}} 02^{\mathrm{s}} .2$ & $-36^{\circ} 16^{\prime} 24^{\prime \prime}$ & $891 \ddagger$ & $9.38 \dagger$ & FIR, H I \\
\hline NGC1436 & 290 & $03^{\mathrm{h}} 43^{\mathrm{m}} 37^{\mathrm{s}} .1$ & $-35^{\circ} 51^{\prime} 11^{\prime \prime}$ & $1388^{\diamond}$ & $10.1 \dagger$ & FIR, H I \\
\hline FCC 302 & 302 & $03^{\mathrm{h}} 45^{\mathrm{m}} 12^{\mathrm{s}} .1$ & $-35^{\circ} 34^{\prime} 15^{\prime \prime}$ & $816 \ddagger$ & $8.48_{-0.07}^{+0.09} *$ & $\mathrm{HI}$ \\
\hline FCC306 & 306 & $03^{\mathrm{h}} 45^{\mathrm{m}} 45^{\mathrm{s}} .4$ & $-36^{\circ} 20^{\prime} 48^{\prime \prime}$ & $891 \ddagger$ & $8.68 \dagger$ & FIR, H I \\
\hline NGC1437B & 308 & $03^{\mathrm{h}} 45^{\mathrm{m}} 54^{\mathrm{s}} .8$ & $-36^{\circ} 21^{\prime} 25^{\prime \prime}$ & $1515 \ddagger$ & $9.39 \dagger$ & FIR, H I \\
\hline ESO358-G063 & 312 & $03^{\mathrm{h}} 46^{\mathrm{m}} 19^{\mathrm{s}} .0$ & $-34^{\circ} 56^{\prime} 37^{\prime \prime}$ & $1920 \ddagger$ & $10.04 \dagger$ & FIR, H I \\
\hline FCC316 & 316 & $03^{\mathrm{h}} 47^{\mathrm{m}} 01^{\mathrm{s}} .5$ & $-36^{\circ} 26^{\prime} 15^{\prime \prime}$ & $1547^{\diamond}$ & $8.64_{-0.12}^{+0.07} *$ & FIR \\
\hline FCC 332 & 332 & $03^{\mathrm{h}} 49^{\mathrm{m}} 49^{\mathrm{s}} .0$ & $-35^{\circ} 56^{\prime} 44^{\prime \prime}$ & $1327^{\diamond}$ & $8.63 \dagger$ & FIR \\
\hline ESO359-G002 & 335 & $03^{\mathrm{h}} 50^{\mathrm{m}} 36^{\mathrm{s}} .7$ & $-35^{\circ} 54^{\prime} 34^{\prime \prime}$ & $1431^{\diamond}$ & $9.21 \dagger$ & FIR \\
\hline
\end{tabular}

1: Common name of the galaxy; 2: Fornax Cluster Catalogue number of the galaxy; 3: right ascension; 4: declination; 5: velocity (defined as the object's redshift times the speed of light); 6: stellar mass. $*$ Stellar masses derived from $3.6 \mu \mathrm{m}$ images (see Section 4.3); †stellar masses from Fuller et al. (2014); łsee Section 3.4; ${ }^{\diamond}$ redshifts from NASA/IPAC Extragalactic Database. 7: Whether the galaxy was selected based on an H I (Waugh et al. 2002; Loni et al. in prep.; based on ATCA data) or FIR (Fuller et al. 2014) detection (or both).

Exceptions are the dwarf galaxies FCC207 and FCC261, for which channel widths of $2 \mathrm{~km} \mathrm{~s}^{-1}$ were used, because of their narrow line widths (see Table 3). The result is a three-dimensional RA-Decvelocity data cube for each galaxy. Primary beam (PB) corrections are then carried out as a separate step using the impbcor command, allowing us to store both PB-corrected and non-PB-corrected data cubes. Beam sizes and sensitivities are listed in Table 3. Typical rms noise levels are around $\sim 3 \mathrm{mJy}_{\text {beam }}{ }^{-1}$. Channel maps of all galaxies in the sample can be found in Appendix D, which is available online.

\subsubsection{NGC1365}

In order to expand our sample, an already reduced image of NGC1365 was taken from the ALMA archive (project ID:
2015.1.01135.S, PI: Egusa, Fumi). It was observed on 2016 March 20. ALMA's $12 \mathrm{~m}$ configuration was used, with a primary beam size of $\sim 55 \mathrm{arcsec}$ at $\sim 115 \mathrm{GHz}$. The mosaic covers an area of $\sim 6.6 \times$ $\sim 4.4$ arcmin. The central frequency of spectral window 3 [the window centred on the ${ }^{12} \mathrm{CO}(1-0)$ line] is $114.848 \mathrm{GHz}$ or $1100 \mathrm{~km} \mathrm{~s}^{-1}$. The bandwidths are $1.875 \mathrm{GHz}$, covering 3840 channels. The spectral resolution is $2.55 \mathrm{~km} \mathrm{~s}^{-1}$. To obtain the final data cube, the CLEAN algorithm in CASA version 4.7.0 was used. A continuum was estimated and subtracted from the channels containing the $\mathrm{CO}$ line as described in Section 3.1.1. A Briggs weighting scheme was adopted (Briggs 1995) with a robust parameter of 0.5. The pixel sizes of the final data cube are 0.3 arcsec, and the channel width is $5 \mathrm{~km} \mathrm{~s}^{-1}$. The synthesized beam size and the sensitivity achieved are listed in Table 3.

Aside from the data reduction, this observation is treated the same as the galaxies observed as part of this survey. 


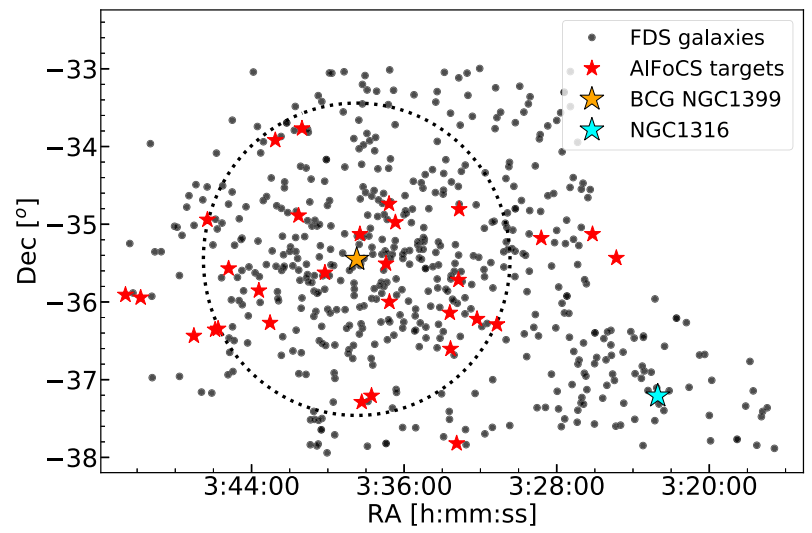

Figure 1. Map of the Fornax cluster. The black dots represent Fornax Deep Survey galaxies from Venhola et al. (2018; see Section 3.3), and the red stars represent the AlFoCS sample. The central galaxy, NGC1399, is indicated with a larger yellow star, and the virial radius (located at $0.7 \mathrm{Mpc}$; Drinkwater, Gregg \& Colless 2001) is shown as a dotted line. AlFoCS targets are distributed evenly over the cluster (except for the infalling subgroup, which was not covered by Herschel). NGC1316, the central galaxy of the infalling subgroup, is indicated with a cyan star.

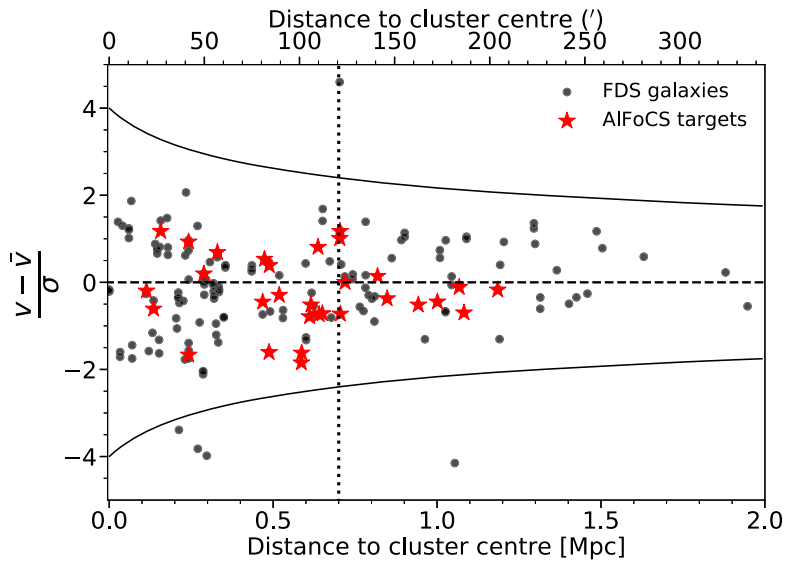

Figure 2. Caustic diagram of the Fornax cluster. The black data points represent the FDS galaxies for which velocity information is available, and the red stars represent the AlFoCS targets. The solid lines represent the escape velocities in the cluster as a function of distance from the cluster centre. The vertical dotted line indicates the virial radius at $0.7 \mathrm{Mpc}$ (Drinkwater et al. 2001). $\bar{v}=1493 \mathrm{~km} \mathrm{~s}^{-1}$ and $\sigma=374 \mathrm{~km} \mathrm{~s}^{-1}$ (Drinkwater et al. 2001). The AlFoCS targets are distributed evenly in the caustic space.

\subsection{Mopra data}

Additional single-dish observations of Fornax cluster galaxies from the Mopra Fornax Cluster CO-Line Legacy Survey (PI: M.W.L. Smith) are included, a survey of ${ }^{12} \mathrm{CO}(1-0)$ in 28 galaxies in the Fornax cluster, carried out between the nights of 2012-08-08 and 2012-09-17. The Mopra Spectrometer (MOPS) was used in wideband mode, centred at a rest frequency of $115.500 \mathrm{GHz}$ for all targets. Its coverage is $8.3 \mathrm{GHz}$ (or $30378 \mathrm{~km} \mathrm{~s}^{-1}$ ), and its spectral resolution is $0.915 \mathrm{~km} \mathrm{~s}^{-1}$. The full width at half-maximum (FWHM) of the beam is $33 \pm 2 \operatorname{arcsec}$ at $115 \mathrm{GHz}$ (Ladd et al. 2005). The calibration uncertainty is less than 10 percent (Ladd et al. 2005); we adopt a conservative value of 10 per cent here. The data were reduced using the ATNF LIVEDATA (Barnes et al. 2001) and GRIDZILLA (Sault, Teuben \& Wright 1995) packages. LIVEDATA is used to fit baselines and transform the raw data files to SDFITS files. We fit linear baselines to all spectra and mask the top and bottom 300 channels. GRIDZILLA is then used to combine these files into data cubes. The spatial resolution of these cubes is 0.25 arcmin per pixel. We use our own scripts to combine the data from the various pointings into a mosaic. For a few objects only single pointings were required. For these objects the data reduction is done using our own scripts to obtain the quotient spectrum by subtracting and dividing by the obtained reference spectra, performing baseline subtraction, and velocity-binning the data. A ripple in the baseline is present in some of the data. This is a known issue with the Mopra telescope, and attempts to mitigate it here, for example by flagging in Fourier space, were not successful. The noise levels in these data are higher, but the data are still usable for the aims of this work.

\subsection{Optical data}

To allow for a comparison of the distribution of the cold molecular gas with the stellar bodies of the galaxies, and to create three-colour images, $r-, g_{-}$, and $u$-band images were obtained from the FDS (Iodice et al. 2016, 2017; Venhola et al. 2017, 2018; Peletier et al., in prep.) for all galaxies in which $\mathrm{CO}(1-0)$ was detected in AlFoCS. The FDS is a new, deep multiband optical survey of the Fornax cluster, which covers 26 square degrees around the virial radius, including the SW subgroup centred on NGC1316 (Iodice et al. 2017). It has been obtained with the ESO VLT Survey Telescope (VST), which is a 2.6-meter-diameter optical survey telescope located at Cerro Paranal, Chile (Schipani et al. 2012). The imaging is done in the $u^{\prime}$, $g^{\prime}, r^{\prime}$, and $i^{\prime}$ bands using the $1^{\circ} \times 1^{\circ}$ field-of-view OmegaCAM instrument (Kuijken et al. 2002) attached to the VST. The deep images provide data with excellent resolution with a mean seeing of 1 arcsec and pixel size of 0.2 arcsec. The quality of the data and the photometry of the galaxies are described in detail in Venhola et al. (2018). The survey area is covered with homogeneous depth with the $1 \sigma$ limiting surface brightness over 1 pixel area of 26.6, 26.7, 26.1, and $25.5 \mathrm{mag} \operatorname{arcsec}^{-2}$ in $u^{\prime}, g^{\prime}, r^{\prime}$, and $i^{\prime}$, respectively. When averaged over a $1 \operatorname{arcsec}^{2}$ area, these numbers correspond to 28.3, 28.4, 27.8, $27.2 \mathrm{mag} \mathrm{arcsec}^{-2}$ in $u^{\prime}, g^{\prime}, r^{\prime}$, and $i^{\prime}$, respectively. The photometric calibration errors of the FDS are 0.04, 0.03, 0.03, and 0.04 mag in $u^{\prime}, g^{\prime}, r^{\prime}$, and $i^{\prime}$, respectively. Venhola et al. (2018) produced Sérsic model fits for all the dwarf galaxies within the survey area using GALFIT (Peng et al. 2002, 2010). In addition, Iodice et al. (2018) have studied all bright $\left(m_{\mathrm{B}}<15 \mathrm{mag}\right)$ early-type galaxies inside the virial radius of the cluster (some of them are presented in this work). They released the total magnitudes, effective radii, and stellar masses and discussed the structure and colours of the galaxy outskirts.

\subsection{Redshift determinations}

A subset of the AlFoCS objects were observed with the $3.9 \mathrm{~m}$ AngloAustralian Telescope at the Siding Spring Observatory as part of a larger programme. The AAOmega spectrograph (Saunders et al. 2004; Sharp et al. 2006) and the Two-degree Field (2dF; Lewis et al. 2002) fibre positioner were used, along with the $580 \mathrm{~V}$ and $385 \mathrm{R}$ gratings, providing spectral coverage over $3740-8850 \AA$. The spectra were reduced using the 2DFDR software package (Croom, Saunders \& Heald 2004), and spectral classifications and redshifts were determined using marz (Hinton et al. 2016). Velocities derived from these redshifts are listed in Table 1, indicated with a $\ddagger$.

\subsection{Moment maps}

Cleaned data cubes were used to produce moment maps of the $\mathrm{CO}(1-0)$ line emission, using the masked-moment method (Dame 
Table 2. Observational parameters.

\begin{tabular}{|c|c|c|c|c|c|c|c|c|c|}
\hline $\begin{array}{l}\text { SB } \\
- \\
(1)\end{array}$ & $\begin{array}{c}\text { Date } \\
- \\
(2)\end{array}$ & $\begin{array}{c}\text { \# ants } \\
- \\
(3)\end{array}$ & $\begin{array}{c}\text { TOT } \\
(\mathrm{min}) \\
(4)\end{array}$ & $\begin{array}{c}\text { Bandpass cal. } \\
- \\
\text { (5) }\end{array}$ & $\begin{array}{c}\text { Flux cal. } \\
- \\
\text { (6) }\end{array}$ & $\begin{array}{c}\text { CF spw } 3 \\
(\mathrm{GHz}) \\
(7)\end{array}$ & $\begin{array}{c}\text { CV spw } 3 \\
\left(\mathrm{~km} \mathrm{~s}^{-1}\right) \\
(8)\end{array}$ & $\begin{array}{c}\text { BW spw } 3 \\
\left(\mathrm{~km} \mathrm{~s}^{-1}\right) \\
(9)\end{array}$ & $\begin{array}{c}\text { CF spws } 0,1,2 \\
\text { (GHz, resp.) } \\
\text { (10) }\end{array}$ \\
\hline Single fields & 07-01-2016 & 42 & 52 & J0336-3616 & J0336-3616 & 114.547 & 1885 & 4898 & $113.001,100.939,102.544$ \\
\hline Small mosaics & $11-01-2016$ & 46 & 125 & Uranus & J0336-3616 & 114.756 & 1340 & 4907 & $112.818,100.824,102.713$ \\
\hline Dwarfs & $12-01-2016$ & 43 & 251 & Uranus & J0336-3616 & 114.716 & 1445 & 4900 & $113.161,101.089,102.703$ \\
\hline
\end{tabular}

1: Scheduling Block; 2: date of the observations; 3: number of antennas used; 4: total observation time in minutes; 5: bandpass calibrator; 6: flux calibrator; 7: central frequency of spectral window 3 [centred on the ${ }^{12} \mathrm{CO}(1-0)$ line]; 8: central velocity of spectral window 3 [centred on the ${ }^{12} \mathrm{CO}(1-0)$ line]; 9: bandwidth of spectral window 3 [centred on the ${ }^{12} \mathrm{CO}(1-0)$ line]; 10 : central frequencies of the remaining spectral windows.

Table 3. Observed and derived properties of the AlFoCS targets.

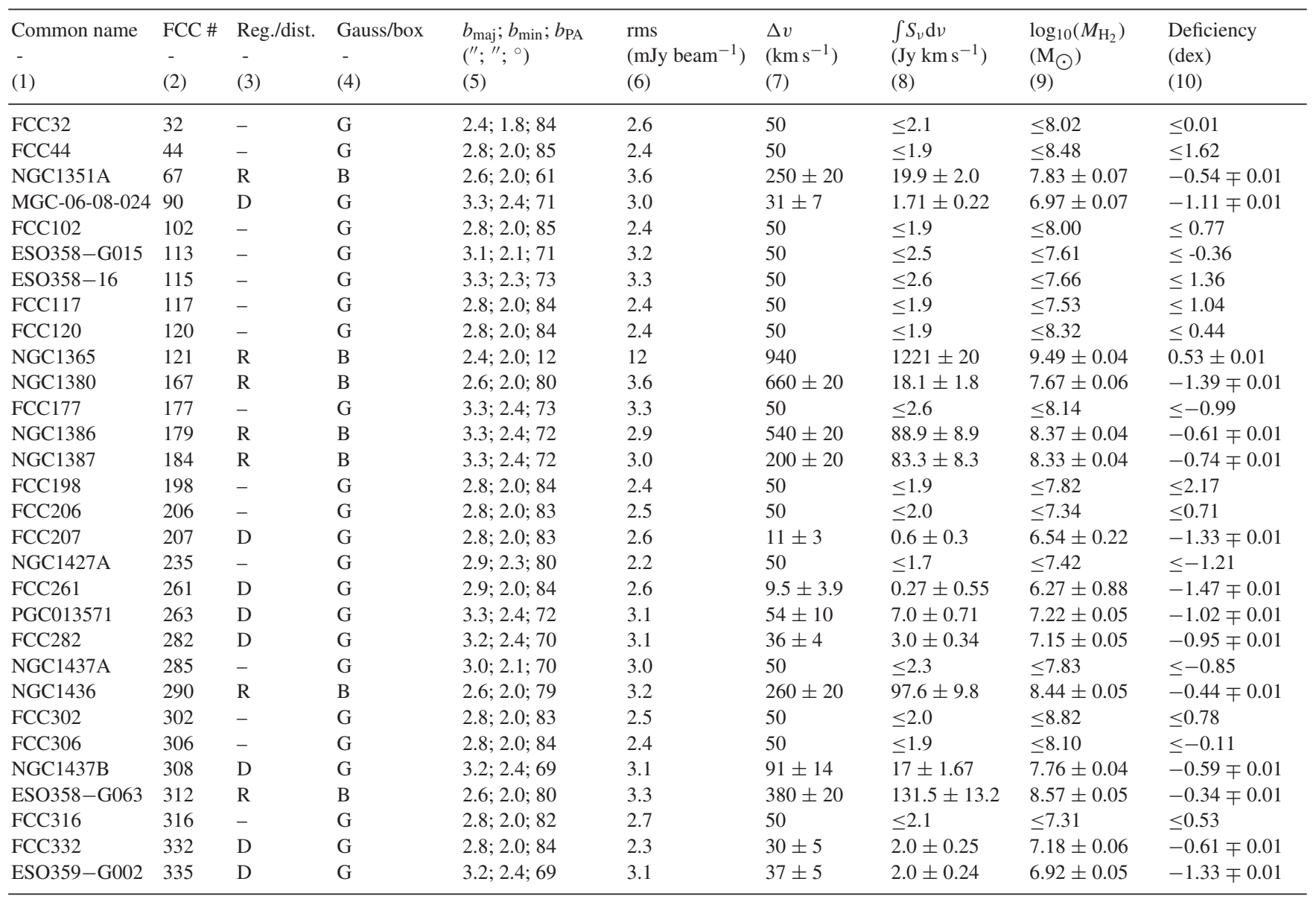

1: Common name of the galaxy; 2: Fornax Cluster Catalogue number of the galaxy; 3: whether the morphology and kinematics of the molecular gas in the galaxy are regular (R) or disturbed (D) (see Section 5.1); 4: whether the line profile of the $\mathrm{CO}(1-0)$ line is best described by a Gaussian (G) or a boxy (B) profile (see Section 4.3); 4/7: upper limits were determined assuming a Gaussian line profile with a full width at half-maximum of $50 \mathrm{~km} \mathrm{~s}^{-1}$ (see Section 4.3 .1 ); 5: beam major axis, minor axis, and position angle; 6: the typical rms in a single channel in the line-free channels of the data cube; 7: the width of the CO integrated spectrum (see Section 4.3); 8: the total CO emission; 9: total $M_{\mathrm{H}_{2}}$ mass derived from the CO emission (see Section 4.3); 10: $\mathrm{H}_{2}$ deficiency, defined as $M_{\mathrm{H}_{2}, \text { observed }}-M_{\mathrm{H}_{2} \text {,expected }}$ (see Section 4.3).

2011). While PB-corrected images are used in the remainder of this work, for the purpose of clarity uncorrected maps are presented in Figs 3 and 4 and Appendix B. In order to create the mask, a Gaussian smoothing was applied to a copy of the data cube, in both spatial axes as well as the velocity axis, with an FWHM of 1.5 times the beam's major axis for the spatial axes and 4 channels (proven to be optimal from previous experience) for the velocity axis. Using this smoothed copy as a mask, the data cubes were then 'clipped' to the $x \sigma$ level, which means that all spaxels below this value are set to zero, where $x$ is chosen to give the best visual result, and equals 3 or 4 .
In Figs 3 and 4 moment maps of NGC1387 and MCG-06-08-024 are shown, serving as examples of the regular and disturbed galaxies, respectively (see Section 5.1 for more details). The top left panel of each of these figures is a three-colour image, constructed using the $r$-, $g$-, and $u$-band images from the Fornax Deep Survey (Iodice et al. 2016, 2017; Venhola et al. 2017, 2018; Peletier et al., in prep.; see Section 3.3). The top right panels are intensity or moment zero maps of the cold molecular gas as traced by the ALMA CO data, showing its spatial distribution. The black ellipse in the lower left corner shows the beam of the observations, and a $1 \mathrm{kpc}$ scale bar is shown 


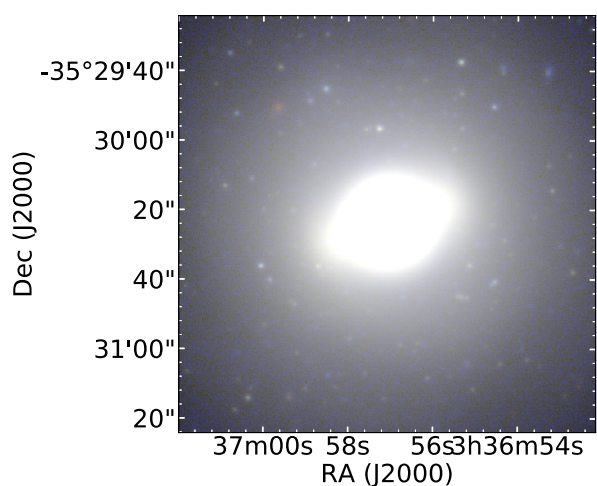

(a)

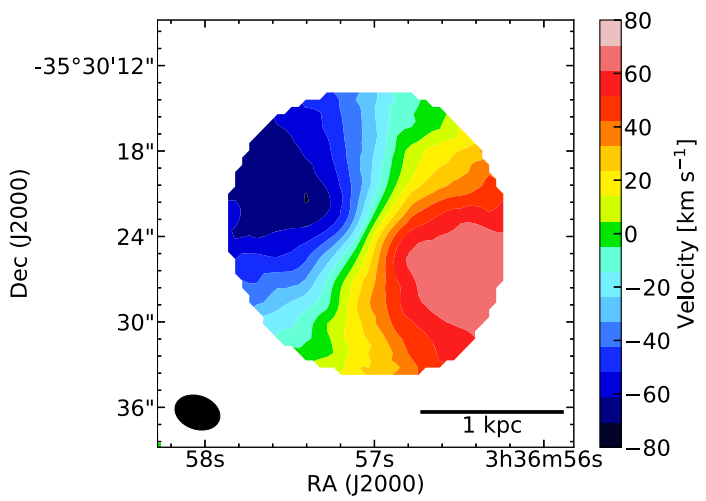

(c)

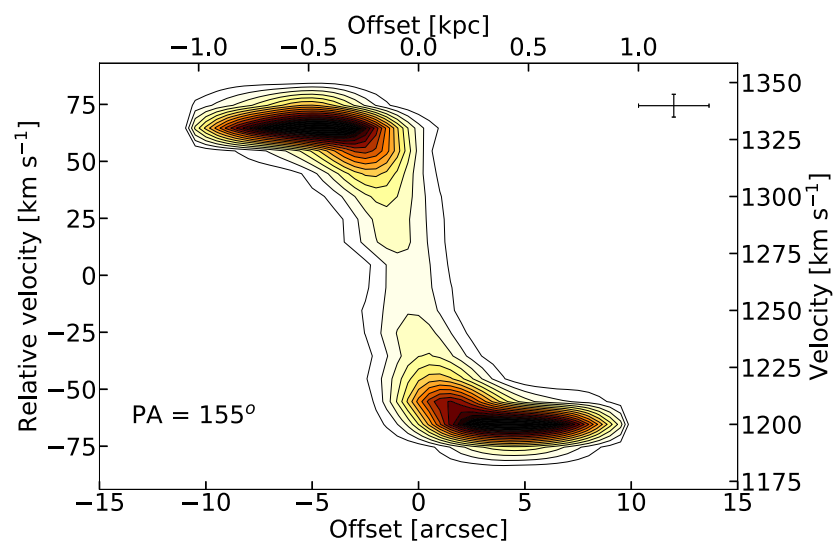

(e)

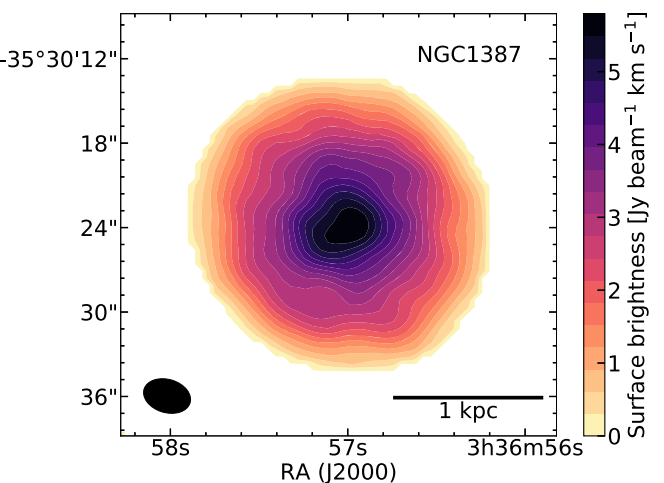

(b)

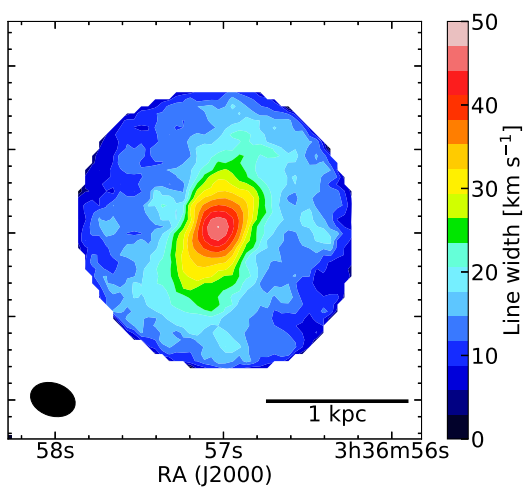

(d) in the lower right corner. This is the same in the other two moment maps. The middle left panels are velocity or moment one maps of the galaxies. Each of the colours represents a $10 \mathrm{~km} \mathrm{~s}^{-1}\left(2 \mathrm{~km} \mathrm{~s}^{-1}\right.$ for FCC207 and FCC261; see Section 3.1.1) velocity channel. The warm colours represent the positive, redshifted velocities, and the cold colours represent the negative, blueshifted velocities. Middle right figures are moment two maps, representing the line width.

The bottom left figures are PVDs, which reveal the motion of gas along the major axes of the galaxies. They are obtained by defining a slit the size of the beam along the major axis of the galaxy in the data cube, and collapsing it along the minor axis. The errorbars in the upper right corner indicate the point spread function FWHM (horizontal) and channel width (vertical). The bottom right figures show the part of the galaxy's spectrum containing the $\mathrm{CO}(1-0)$ line. The spectrum was obtained by defining a rectangular aperture around the detected emission in the spatial directions, large enough to contain all its $\mathrm{CO}$ emission, and then collapsing the data cube along both spatial axes.

In NGC1387 (Fig. 3b) the gas is distributed as an almost face-on disc, with the projected intensity decreasing radially. Its velocities 


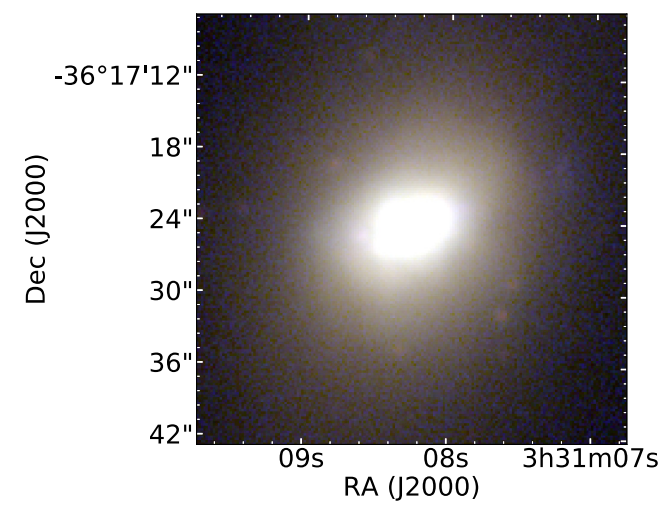

(a)

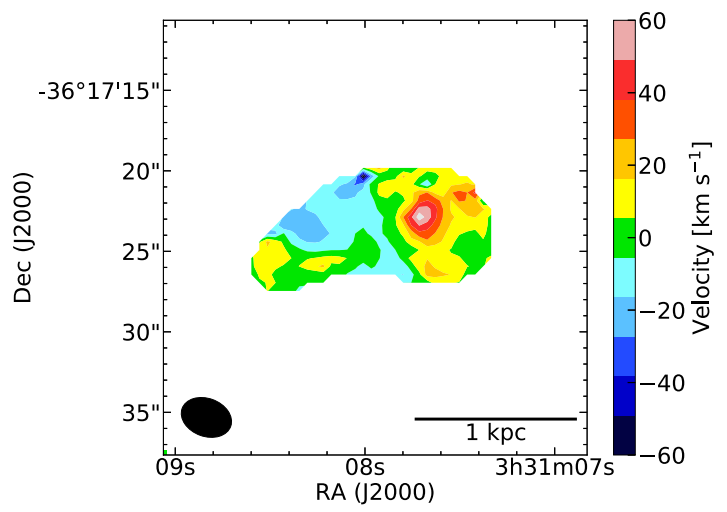

(c)

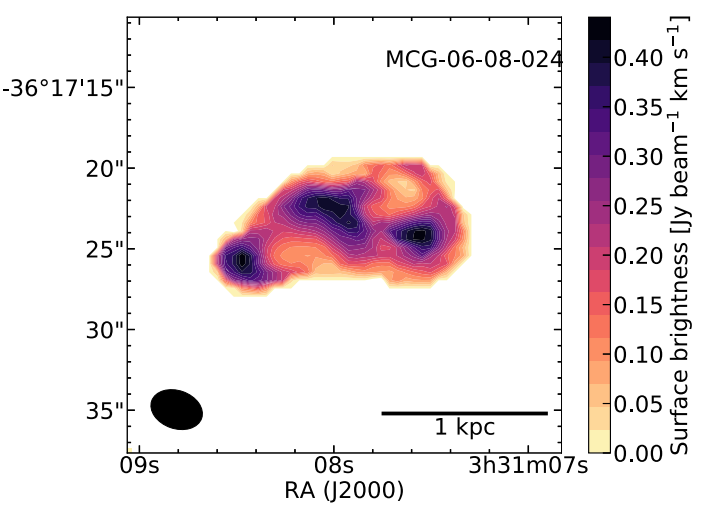

(b)

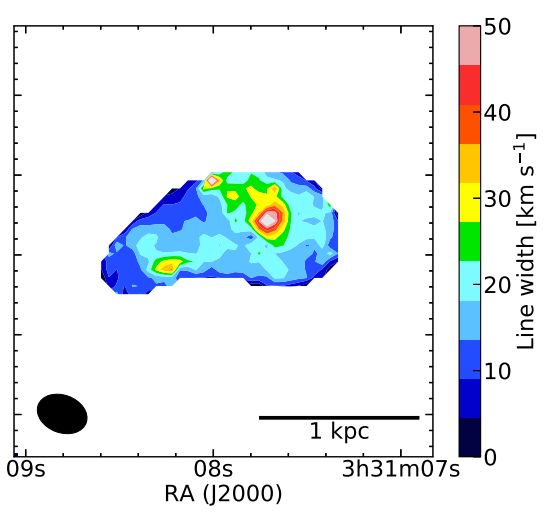

(d)

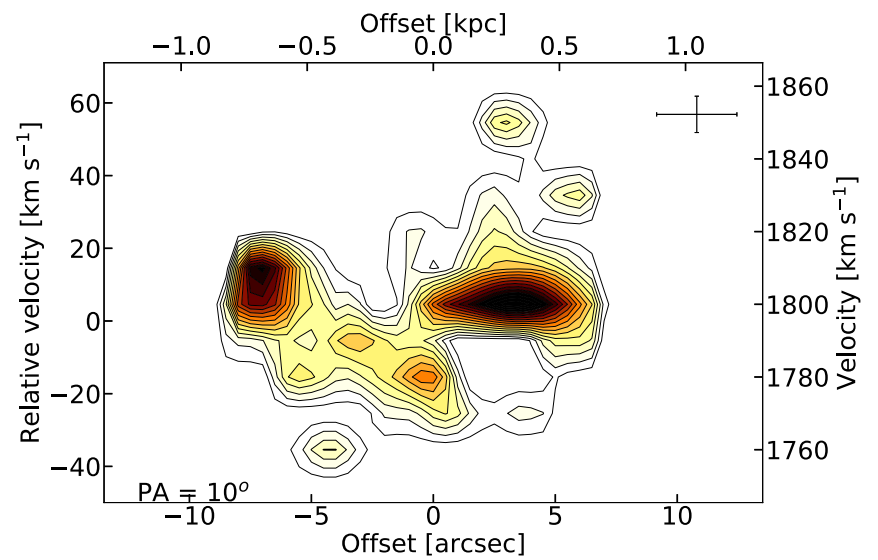

(e)

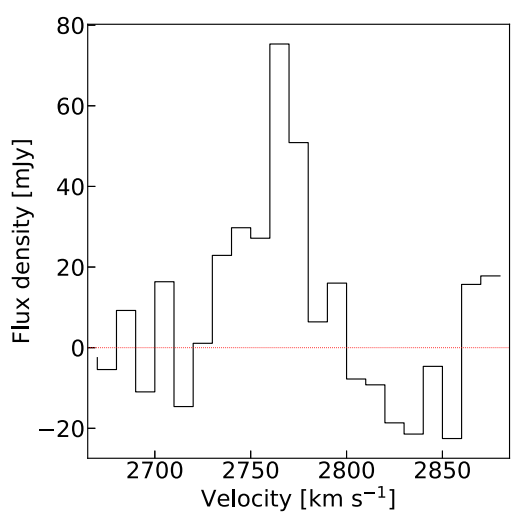

(f)

Figure 4. MGC-06-08-024 (FCC90), similar to Fig. 3. MGC-06-08-024 is a galaxy with irregular CO emission, and therefore has irregular moment maps and an irregular position-velocity diagram.

vary between -80 and $+80 \mathrm{~km} \mathrm{~s}^{-1}$ relative to the systemic velocity, which is determined by taking the mean of the moment one map shown here. The line is widest in a band along the kinematic minor axis, due to beam-smeared rotation. The PVD of NGC1387 (Fig. 3e) is very regular, showing a smooth and symmetric 'rotation curve', which reaches its maximum very quickly. The doublepeaked line profile, typical for a disc, is clearly visible in its spectrum (Fig. 3f).

In MCG-06-08-024 the molecular gas is distributed irregularly, around three different maxima. The velocities of the gas are between -60 and $+60 \mathrm{~km} \mathrm{~s}^{-1}$ relative to the systemic velocity. The PVD of
MCG-06-08-024 has a very irregular shape. Similar images of the remaining 14 detected galaxies were created in the same way, and can be found in Appendix B, which is available online.

\subsection{Comparison to optical morphology}

Fig. 5 overplots the $\mathrm{CO}$ integrated intensity contours on top of optical images of the galaxies ( $g$-band images of the FDS were used; see Section 3.3). The CO emission is shown as 10 coloured contours, the outer contour being equal to $3-4 \sigma$, while the innermost contour depends on the highest signal measured in the galaxy in 


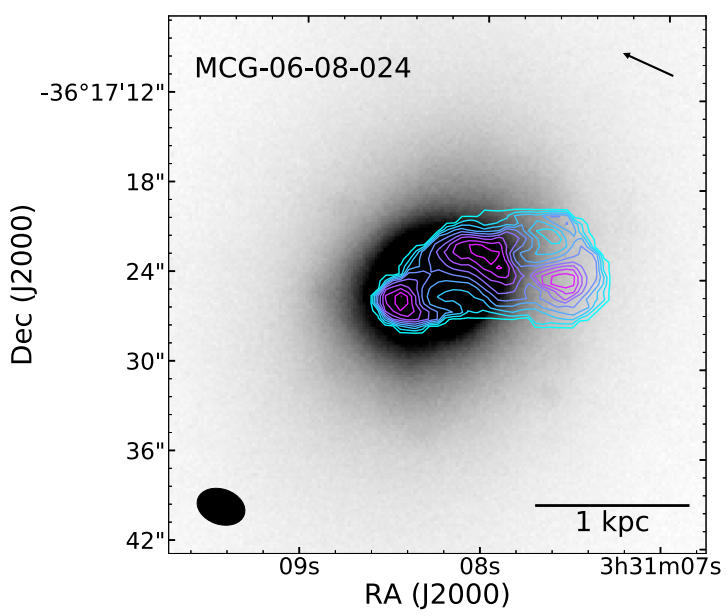

(a)

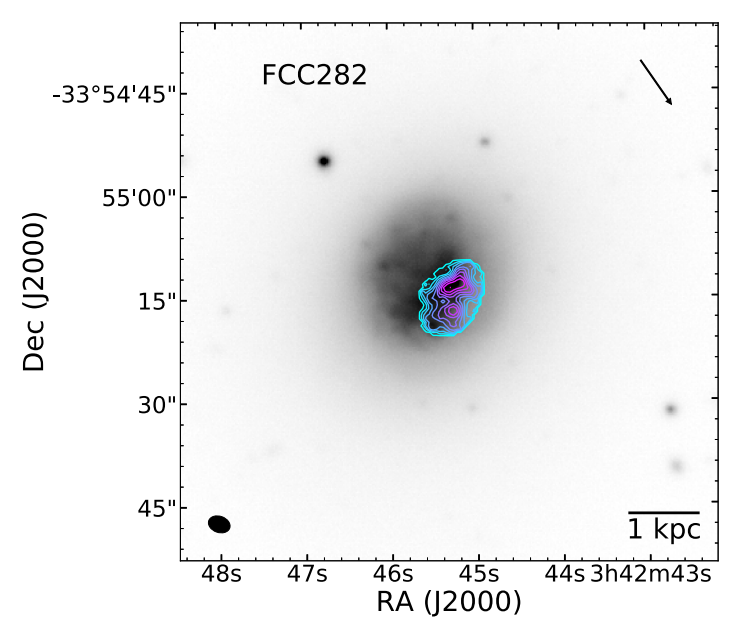

(c)

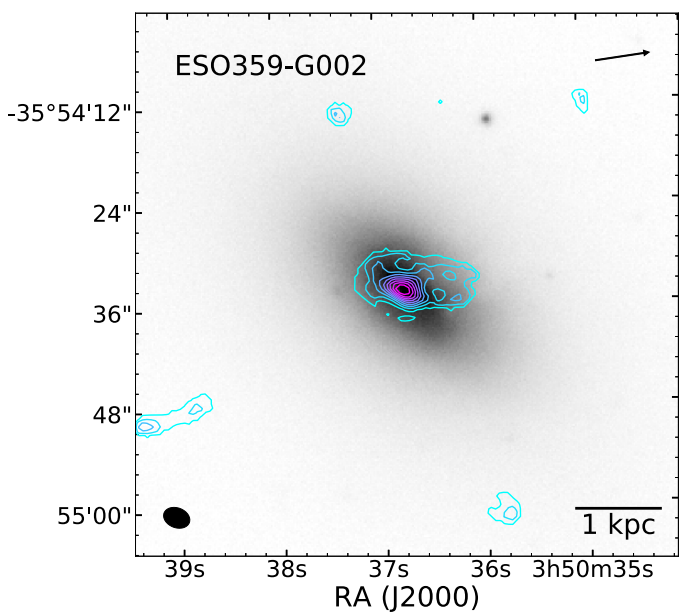

(b)

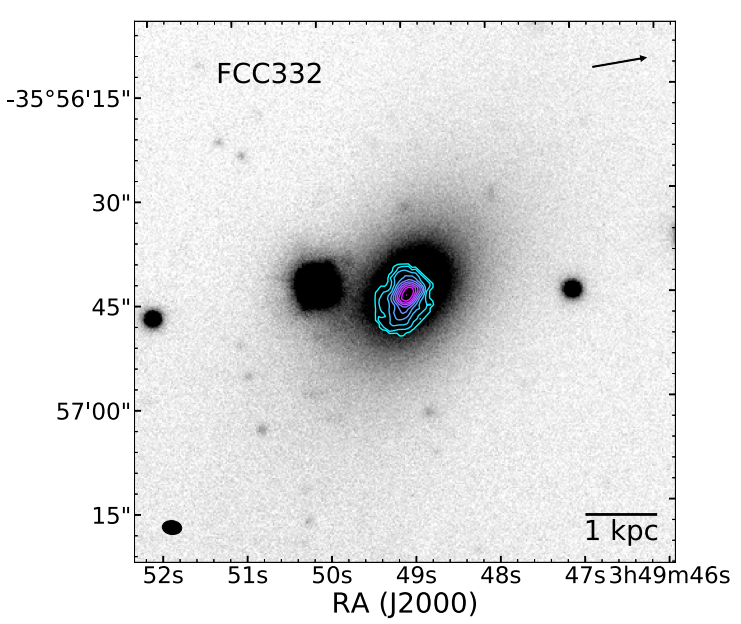

(d)

Figure 5. CO(1-0) emission overplotted on optical ( $g$-band) images from the FDS (Iodice et al. 2016, 2017; Venhola et al. 2017, 2018; Peletier et al., in prep.). The $\mathrm{CO}$ emission is shown as 10 coloured contours; the outer contour is set equal to 3 or $4 \sigma$, while the level of the innermost contour depends on the highest signal measured in the galaxy. The beam is shown in the lower left corners, and a $1 \mathrm{kpc}$ scale bar in the lower right corners. The arrow in the upper right corners indicates the direction towards the cluster centre. The molecular gas in these galaxies is asymmetric with respect to their stellar bodies. In (a) and (b) it extends beyond the stellar body and forms a tail aligned with the direction of the cluster centre.

question. The arrows in the upper right corners point towards the cluster centre (here defined as the location of the BCG NGC1399). Similar plots for the remaining galaxies can be found in Appendix $\mathrm{C}$, which is available online.

The galaxies in Fig. 5 are all examples of galaxies with irregular CO emission, asymmetric compared to the galaxy's stellar body. In MCG-06-08-024 (Fig. 5a) and ESO359-G002 (Fig. 5b) the molecular gas forms a tail that extends beyond the stellar body. These galaxies are discussed further in Section 5.3. Other examples of galaxies with asymmetric CO emission are FCC207 (Fig. C0g) and FCC261 (Fig. COh). In the cases of the regular galaxies, such as ESO358-G063 (Fig. C1m), NGC1386 (Fig. C1e), NGC1387 (Fig. C1f), and NGC1351A (Fig. C1a), the CO emission follows the optical shape of the galaxy. The CO emission in NGC1380 (Fig. C1d) is very compact compared to its stellar body in our images, but has been shown to be distributed in a regular disc by Boizelle et al. (2017).
It would be interesting to compare the $\mathrm{CO}$ morphologies to $\mathrm{HI}$ morphologies, especially for the galaxies that exhibit asymmetric $\mathrm{CO}$ emission or gas tails. This would show us whether these galaxies also have $\mathrm{HI}$ tails, which is expected if ram pressure stripping is at play. The current $\mathrm{HI}$ observations available are not of sufficient resolution to do this. However, in the future we will be able to use data from the MeerKAT Fornax Survey for this purpose.

\section{RESULTS}

$\mathrm{CO}$ was detected (at $>3 \sigma$ ) in 15 of the 30 galaxies observed. In Fig. 6 the (projected) locations of the detections and non-detections within the cluster are shown, and morphologically and kinematically regular and disturbed galaxies are highlighted. All FDS (see Section 3.3) galaxies are shown as black dots, and the AlFoCS galaxies are shown in colour. Non-detections are shown as blue plus signs, the pink squares are galaxies in which $\mathrm{CO}$ is detected 


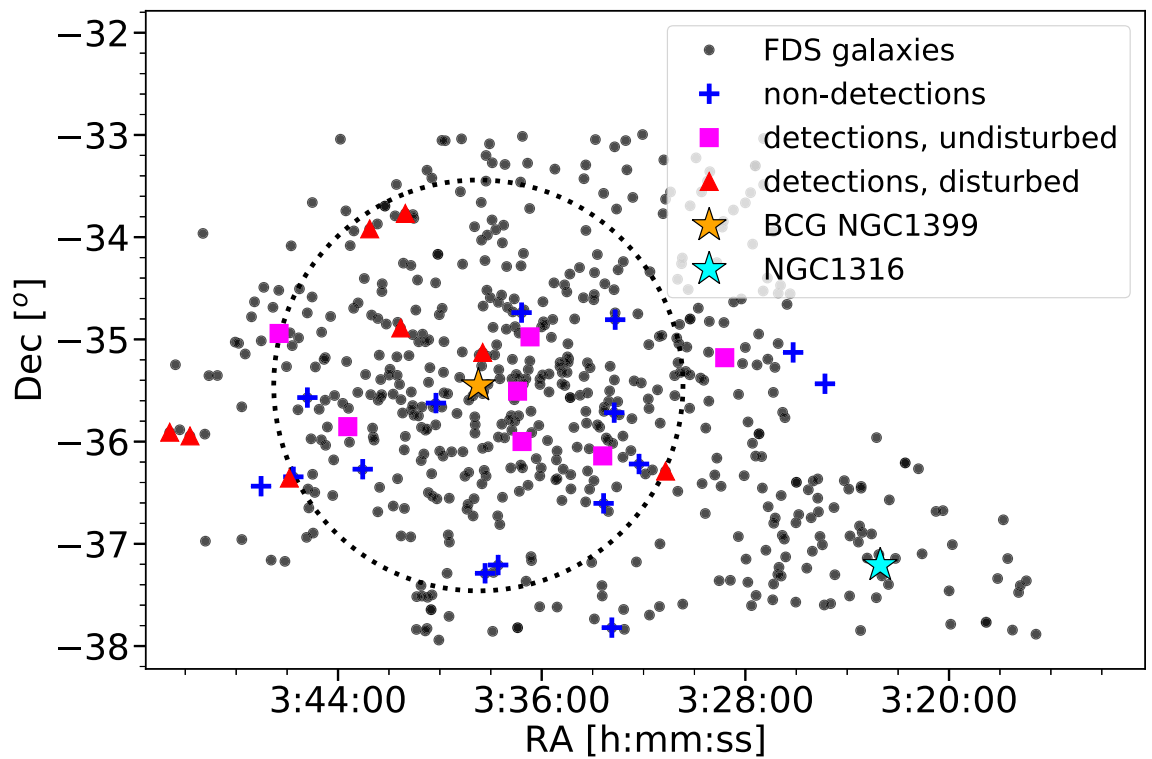

Figure 6. Map of the Fornax cluster, similar to Fig. 1. The coloured symbols represent the AlFoCS targets; their shape and colour indicate whether CO was detected and if so, whether it is morphologically and kinematically disturbed or regular, as indicated in the legend. The central galaxy, NGC1399, is indicated with a yellow star and the virial radius with a dotted line. The central galaxy of the infalling group in the lower right corner of the figure, NGC1316, is indicated with a cyan star. Non-detections, disturbed galaxies, and regular galaxies are distributed evenly over the cluster.

and morphologically and kinematically regular or undisturbed, and the red triangles represent galaxies in which $\mathrm{CO}$ is detected and morphologically and kinematically disturbed (see Section 5.1 for more details). Both detections and non-detections are distributed evenly over the cluster. At first glance it looks like there are slightly more non-detections south of the cluster centre; however, this is not statistically significant. Galaxies with disturbed molecular gas reservoirs seem to be mainly located close to or outside the virial radius.

\subsection{Marginal detections}

In ESO358-G015, FCC32, and NGC1437A CO is detected, but only marginally. In ESO358-G015 and NGC1437A these are 4$5 \sigma$ detections, but the emission comes from small features away from the galactic centre, and it is not clear whether this emission is related to the galaxy observed. For FCC 32 we find a tentative $2 \sigma$ peak at the centre of the galaxy. These features are likely noise, and for these reasons we do not consider these observations further in this work.

\subsection{Continuum detections}

Continuum (3 mm) was detected in NGC1380, NGC1386, NGC1387, and NGC1427A. In Fig. 7 the continuum maps of NGC1380, NGC1386, and NGC1387 are shown as coloured contours overplotted on the $g$-band images from the FDS, similar to Fig. 5. In all three cases the continuum emission originates from the galactic centre. Two galaxies, NGC1380 and NGC1386, are known to harbour active galactic nuclei (AGNs; e.g. Lena et al. 2015; Boizelle et al. 2017; Rodríguez-Ardila et al. 2017). The emission we detect is an unresolved point source at the galactic centre, but has a positive spectral index (see Table 4). It is possible that both thermal emission and non-thermal emission are contributing the observed emission in these sources.
The $3 \mathrm{~mm}$ continuum emission in NGC1387 has a point-like morphology in the lower sideband, but when imaged at the higher frequencies several additional point sources are also detected, in the region where we know dust and molecular gas are present. This additional emission leads to the very large spectral index measured for this source (see Table 4). Given this, the detected $3 \mathrm{~mm}$ emission is again likely due to a mix of AGN activity and thermal emission from dust.

In the case of NGC1427A the emission originates from a small source at the edge of the galaxy. This is shown and discussed separately in Section 5.5.

\section{3 $\mathrm{H}_{2}$ masses}

$\mathrm{H}_{2}$ masses for all detected galaxies were estimated using the following equation:

$M_{\mathrm{H}_{2}}=2 m_{\mathrm{H}} D^{2} X_{\mathrm{CO}} \frac{\lambda^{2}}{2 k_{\mathrm{B}}} \int S_{v} \mathrm{~d} v$,

where $m_{\mathrm{H}}$ is the mass of a hydrogen atom, $D$ is the distance to the galaxy, $X_{\mathrm{CO}}$ is the $\mathrm{CO}$-to- $\mathrm{H}_{2}$ mass conversion factor, $\lambda$ is the rest wavelength of the line observed, $k_{\mathrm{B}}$ is the Boltzmann constant, and $\int S_{v} \mathrm{~d} v$ is the total flux of the line observed.

We use the metallicity-dependent mass conversion factor derived from Accurso et al. (2017; equation 25):

$$
\begin{array}{r}
\log \alpha_{\mathrm{CO}}=14.752-1.623[12+\log (\mathrm{O} / \mathrm{H})] \\
+0.062 \log \Delta(\mathrm{MS}),
\end{array}
$$

where $12+\log (\mathrm{O} / \mathrm{H})$ is the metallicity and $\log \Delta(\mathrm{MS})$ is the distance from the main sequence, discussed below. The $1 \sigma$ spread in $\log \alpha_{\mathrm{CO}}$ from this relation is $0.165 \mathrm{dex}$. It is multiplied by $2.14 \times 10^{20}$ to obtain $X_{\mathrm{CO}}$ (Bolatto, Wolfire \& Leroy 2013). For reference, this equation gives a conversion factor of $2.08 \pm 0.02 \times 10^{20}$ for solar metallicity $[12+\log (\mathrm{O} / \mathrm{H})=8.69$; Asplund et al. 2009]. Since we do not have independent metallicity measurements for 

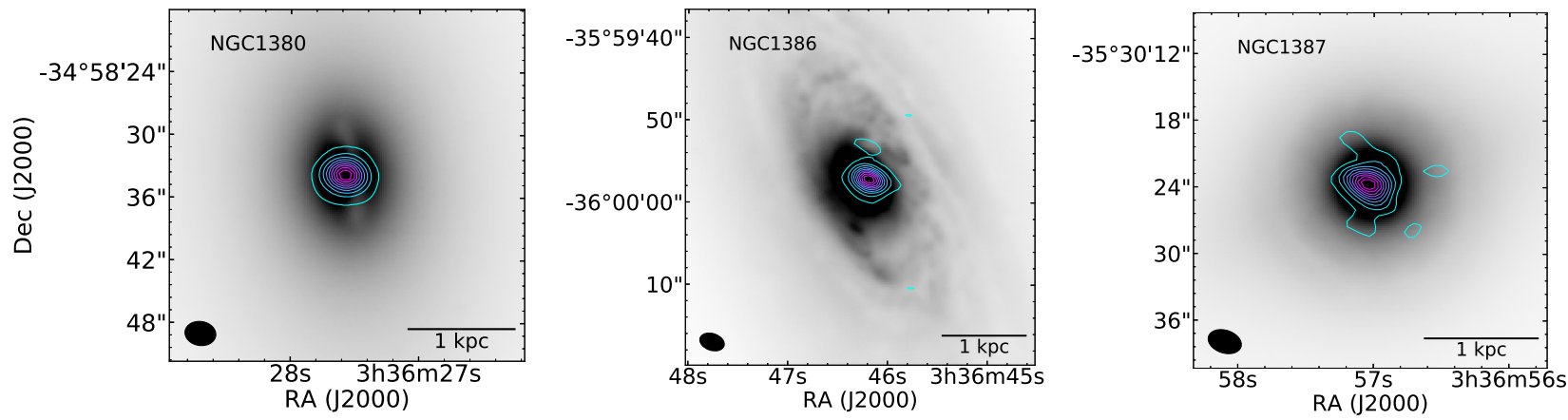

Figure 7. $3 \mathrm{~mm}$ continuum emission overplotted on optical ( $g$-band) images from the FDS (see Section 3.3), similar to Fig. 5. The emission is shown as 10 coloured contours; the lower (outer) contour level equals $5 \sigma$. The emission originates from the galaxies' centres, and is likely due to a combination of AGN activity and thermal emission from dust.

Table 4. Properties of the detected $3 \mathrm{~mm}$ continuum emission.

\begin{tabular}{|c|c|c|c|c|c|c|c|}
\hline $\begin{array}{l}\text { Galaxy } \\
- \\
(1)\end{array}$ & $\begin{array}{l}\text { Frequency } \\
(\mathrm{GHz}) \\
(2)\end{array}$ & $\begin{array}{l}\text { Flux density } \\
(\mathrm{mJy}) \\
(3)\end{array}$ & $\begin{array}{l}\text { Frequency USB } \\
(\mathrm{GHz}) \\
(4)\end{array}$ & $\begin{array}{l}\text { Flux density USB } \\
(\mathrm{mJy}) \\
(5)\end{array}$ & $\begin{array}{l}\text { Frequency LSB } \\
(\mathrm{GHz}) \\
(6)\end{array}$ & $\begin{array}{l}\text { Flux density LSB } \\
\text { (mJy) } \\
(7)\end{array}$ & $\begin{array}{l}\text { Spectral index } \\
- \\
(8)\end{array}$ \\
\hline NGC1386 & 107.718 & $3.69 \pm 0.05$ & 113.750 & $3.99 \pm 0.07$ & 101.748 & $3.64 \pm 0.07$ & $0.8 \pm 0.2$ \\
\hline NGC1387 & 107.718 & $1.85 \pm 0.06$ & 113.750 & $4.3 \pm 0.1$ & 101.748 & $1.04 \pm 0.06$ & $12.7_{-0.5}^{+0.6}$ \\
\hline NGC1427A & 107.765 & $0.16 \pm 0.03$ & 113.763 & $0.20 \pm 0.06$ & 101.775 & $0.16 \pm 0.03$ & $2.0_{-3.5}^{+3.0}$ \\
\hline
\end{tabular}

1: Name of the galaxy; 2: central frequency of the $3 \mathrm{~mm}$ continuum; 3: flux density of the $3 \mathrm{~mm}$ continuum emission; 4: central frequency of the upper sideband (USB); 5: flux density of the continuum in the upper sideband; 6: central frequency of the lower sideband; 7: flux density of the continuum in the lower sideband (LSB); 8: spectral index of the continuum emission.

each object, metallicities were derived directly from the stellar masses of the galaxies, using the mass-metallicity relation from Sánchez et al. (2017), which uses the calibration from Pettini \& Pagel (2004). Stellar masses $\left(M_{\star}\right)$ are listed in Table 1 . They were taken from Fuller et al. (2014) where possible (see Table 1). Alternatively, they were obtained from aperture photometry on archival Wide-field Infrared Survey Explorer (WISE; Wright et al. 2010) band $1(3.6 \mu \mathrm{m})$ images, assuming a mass-to-light ratio of 1 (see Table 1). Apertures were chosen using the effective radii determined by Venhola et al. (2018; see Section 3.3) if available, and alternatively chosen by eye. Uncertainties on the stellar mass in these cases are a combination of the uncertainty in the effective radius and the rms in the image.

The $X_{\mathrm{CO}}$ calibration from Accurso et al. (2017) requires a distance from the main sequence (e.g. Brinchmann et al. 2004; Elbaz et al. 2007; Noeske et al. 2007). Here we assume a distance from the main sequence $\Delta \mathrm{MS}=0$ for all galaxies. It is a second-order parameter, so varying it does not strongly affect our results. Equation 2 is valid for values of $-0.8<\Delta \mathrm{MS}<1.3$. Varying $\Delta \mathrm{MS}$ over this range results in a maximum error of 0.08 in $\alpha_{\mathrm{CO}}$, which is indeed small compared to the other errors.

To make sure we included all the $\mathrm{CO}$ emission, while minimizing the inclusion of noise, galaxies were subdivided into two groups: a group whose line profiles are best described by a Gaussian profile (mostly dwarf galaxies with narrow CO lines) and another group whose line profiles are best described by a box profile (mostly larger galaxies). Which profile best describes a galaxy is listed in Table 3 . The widths of the CO integrated spectra are given. For boxy line profiles an uncertainty of $20 \mathrm{~km} \mathrm{~s}^{-1}$ (the equivalent of two channels) is adopted; for Gaussian profiles the formal fitting errors on the line width are quoted. For the first group we fit a Gaussian to the $\mathrm{CO}(1-0)$ line and integrate this fit to obtain the total line flux. For the second group, we integrate directly under the line observed. In this case the boundaries of the line are determined using the PVDs. Uncertainties are a combination of the error on the total integrated line emission $\int S_{\nu} \mathrm{d} v$ and an adopted 10 percent calibration error, and are often dominated by the latter. For galaxies with a boxy profile, the error in the integrated line emission is estimated according to the following equation, adapted from equation 1 from Young et al. (2011):

$\sigma_{I}^{2}=(\Delta v)^{2} \sigma^{2} N_{l}$,

where $N_{l}$ is the number of channels that is summed over, $\Delta v$ is the width of each channel, and $\sigma$ is the rms noise level in the line free part of the spectrum. For galaxies with an approximately Gaussian line profile, the error on the total integrated line emission is estimated by combining the formal fitting errors on the parameters of the fit. The resulting molecular gas masses are listed in Table 3.

\subsubsection{Upper limits}

For non-detections, $3 \sigma$ upper limits were determined using the rms in the (spatial) inner area of the PB-corrected data cubes. Since all non-detections can be considered dwarf galaxies, we assume Gaussian line profiles with an FWHM of $50 \mathrm{~km} \mathrm{~s}^{-1}$. This is slightly broader than the profiles of the dwarf galaxies detected here, and therefore a conservative assumption. The maximum of the assumed line profile was set to 3 times the rms in the corresponding data cube. We use stellar-mass-dependent $\mathrm{CO}$-to- $\mathrm{H}_{2}$ conversion factors, as described above in Section 4.3. We then use equation 1 to obtain the upper limits for the $\mathrm{H}_{2}$ mass listed in Table 3 . 


\subsubsection{Mopra}

Of the 28 galaxies observed with Mopra, $\mathrm{CO}$ was detected in one additional galaxy which was not observed with ALMA, NGC1317. After removing data affected by bad weather, we were able to obtain this one additional $\mathrm{H}_{2}$ mass measurement and eight additional upper limits. Due to a problem with the observations, we only have single, central pointing observations of NGC1317. Since the molecular gas is usually centrally located, however, we expect this to cover most if not all of its $\mathrm{CO}$ emission. Upper limits are $3 \sigma$ upper limits, estimated as described above. Despite the rather prominent baseline ripple in some of the observations, a known issue with the Mopra Telescope (see Section 3), these upper limits provide reasonably good constraints. The resulting upper limits, as well as the estimated $\mathrm{H}_{2}$ mass of NGC1317, are listed in Table 5.

\subsection{Gas fractions and deficiencies}

In Fig. 8 the galaxies' molecular-to-stellar mass ratios are shown as a function of their stellar mass (see Section 4.3 for more details about the stellar masses used here) and compared with those of field control galaxies with the same stellar masses. The molecular gas fraction is given by $\left(\frac{M_{\mathrm{H}_{2}}}{M_{\text {total }}}\right)$. Since $M_{\mathrm{HI}}$ and $M_{\mathrm{H}_{2}}$ are relatively small contributions to the total mass of the galaxy compared to the stellar mass, for convenience and consistency with the definition in Saintonge et al. (2017) (see below), we define the gas fraction here as $\left(\frac{M_{\mathrm{H}_{2}}}{M_{\star}}\right)$. We use the extended CO Legacy Database for GALEX Arecibo SDSS Survey (xCOLD GASS; Saintonge et al. 2017) as a field galaxy control sample. xCOLD GASS is a survey of molecular gas in the local universe, built upon its predecessor COLD GASS (Saintonge et al. 2011). It is a mass-selected $\left(M_{\star}>10^{9} \mathrm{M}_{\odot}\right)$ survey of galaxies in the redshift interval $0.01<z<0.05$ from the SDSS, and is therefore representative of the local galaxy population within this mass range. We use the relation based on the median values that they obtained by subdividing the sample in bins based on their stellar mass (see Saintonge et al. 2017, their fig. 10) and interpolate linearly (in logspace) to obtain the relation represented by the dashed line. Since xCOLD GASS galaxies were selected to have stellar masses $M_{\star}>10^{9} \mathrm{M}_{\odot}$, the first stellar mass bin is located at $M_{\star}=9.388 \mathrm{M}_{\odot}$. Below this stellar mass, the dashed line is obtained using linear extrapolation (in logspace). Expected mass fractions for galaxies in this mass range, 5 detections and 11 upper limits, should be treated with caution. The shaded areas represent the 1,2 , and $3 \sigma$ levels in the xCOLD GASS data, from dark to lighter. Galaxies with disturbed molecular gas are shown in red, and galaxies with regular, undisturbed molecular gas are shown in black (see Section 5.1 for the definitions). Galaxies that have clear gas tails that extend beyond their optical emission, or otherwise asymmetric CO emission, are indicated with red triangles. ALMA upper limits for the $\mathrm{H}_{2}$ mass are shown as magenta open triangles and Mopra upper limits as cyan open triangles. NGC1317, the only Mopra detection included here, is shown as a cyan dot. There is a systematic offset between the xCOLD GASS $\mathrm{H}_{2}$ mass fractions for field galaxies and our values of up to about $\sim 1$ dex. This offset is not very significant at an individual level for regular galaxies, whose offset is mostly within or close to the $1 \sigma$ scatter in the xCOLD GASS data. With the exception of NGC1437B and FCC332, all disturbed galaxies lie below $3 \sigma$ (for FCC261 and FCC207 we cannot be certain because they lie below the mass range of the xCOLD GASS data, but based on this figure it seems plausible to assume they would fall below $3 \sigma$ as well). In particular the galaxies with asymmetric $\mathrm{CO}$ emission have low gas fractions.
We define $\mathrm{H}_{2}$ deficiencies here as $\log \left(M_{\mathrm{H}_{2} \text {,observed }}\right)$ $\log \left(M_{\mathrm{H}_{2} \text {,expected }}\right)$. Estimates of the $\mathrm{H}_{2}$ deficiency for each galaxy are listed in Table 3. Galaxies with regular $\mathrm{CO}$ emission have an average $\mathrm{H}_{2}$ deficiency of $-0.50 \mathrm{dex}$, and galaxies with disturbed $\mathrm{CO}$ emission have an average $\mathrm{H}_{2}$ deficiency of -1.1 dex. In Fig. 9 $\mathrm{H}_{2}$ deficiencies are plotted as a function of the (projected) distance between the galaxy and the cluster centre. The markers and colours are the same as in Fig. 8. It seems like galaxies within a (projected) radius of $0.4 \mathrm{Mpc}$ from the cluster centre are slightly more deficient than galaxies outside this radius. However, Kolmogorov-Smirnov and Mann-Whitney $U$ tests are unable to reject the null hypothesis that both groups of galaxies are drawn from the same distribution at more than $\sim 2 \sigma$. Possible explanations for this and a further discussion of this figure can be found in Section 5.3.

\section{DISCUSSION}

\subsection{Gas morphologies and kinematics}

The galaxies detected here can be divided into two categories: galaxies with disturbed molecular gas morphologies and regular systems. Whether a galaxy is morphologically disturbed or regular is determined by visual inspection of the moment zero and one maps [see Figs 3 and 4, Appendix B (online), and Table 3]. Non-disturbed galaxies have molecular gas that is concentrated symmetrically around the galactic centre, whereas galaxies with disturbed morphologies contain molecular gas that is asymmetric with respect to the (optical) centre of the galaxy. It sometimes has a very irregular shape, and, in some cases, even extends beyond the galaxy's stellar body (see Section A0.2). Of the galaxies detected here, eight are classified as disturbed galaxies and seven have regular molecular gas morphologies.

The galaxies with morphologically disturbed molecular gas reservoirs also have disturbed molecular gas kinematics. Looking at the velocity maps in Fig. 3 and the regular galaxies in Appendix B (online), regular galaxies follow a standard "spider diagram" shape, indicative of a regular rotation. Disturbed galaxies, on the other hand, have irregular velocity maps, indicating the presence of non-circular motions. In some cases rotation is still present (in NGC1437B and PGC013571, e.g., Figs B12 and B9, respectively); in other cases no rotation can be identified (e.g. ESO359-G002 and FCC332; Figs B15 and B14, respectively). This is also reflected in the PVDs, which look like smooth rotation curves for the regular galaxies but have very asymmetric and irregular shapes for the disturbed galaxies. Maps of the $\mathrm{CO}(1-0)$ line width of the regular galaxies often reveal symmetric structures such as rings and spiral arms (see e.g. Fig B13). For disturbed galaxies this is, again, much more irregular (e.g. Fig. B12). A further discussion of each galaxy in detail can be found in Appendix A.

\subsection{Stripping and gas stirring in Fornax in comparison with the field}

There is a clear mass split between galaxies with regular and disturbed molecular gas morphologies, where all galaxies with stellar masses below $3 \times 10^{9} \mathrm{M}_{\odot}$ have disturbed molecular gas (see Fig. 8). In the absence of a comparable field sample tracing molecular gas at these stellar masses, we compare this result to the Local Irregulars That Trace Luminosity Extremes, The H I Nearby Galaxy Survey (LITTLE THINGS; Hunter et al. 2012). LITTLE THINGS is a multiwavelength survey of 37 dwarf irregular and 4 blue compact nearby ( $\leq 10.3 \mathrm{Mpc}$ ) (field) dwarf galaxies that is centred around 
Table 5. Key properties and derived quantities of the Mopra targets included in this work.

\begin{tabular}{|c|c|c|c|c|c|c|}
\hline $\begin{array}{l}\text { Name } \\
- \\
(1)\end{array}$ & $\begin{array}{l}\text { RA } \\
(\text { J2000) } \\
(2)\end{array}$ & $\begin{array}{l}\text { Dec. } \\
(\mathrm{J} 2000) \\
(3)\end{array}$ & $\begin{array}{l}\text { Stellar mass } \\
\left(\log \left(M_{\odot}\right)\right) \\
(4)\end{array}$ & $\begin{array}{l}\mathrm{rms} \\
\left(\mathrm{mJy} \mathrm{km} \mathrm{s}^{-1}\right) \\
(5)\end{array}$ & $\begin{array}{l}\log _{10}\left(M_{\mathrm{H}_{2}}\right) \\
\left(M_{\odot}\right) \\
(6)\end{array}$ & $\begin{array}{l}\text { Deficiency } \\
(\text { dex }) \\
(7)\end{array}$ \\
\hline NGC1316 & $03^{\mathrm{h}} 22^{\mathrm{m}} 41^{\mathrm{s}} .718$ & $-37^{\circ} 12^{\prime} 29^{\prime \prime} 62$ & $10.0 \dagger$ & 273 & $\leq 8.27$ & $\leq-0.52$ \\
\hline NGC1317 & $03^{\mathrm{h}} 22^{\mathrm{m}} 44^{\mathrm{s}} .286$ & $-37^{\circ} 06^{\prime} 13^{\prime \prime} .28$ & $9.98 \dagger$ & 192 & $8.69 \pm 0.04$ & $-0.08 \pm 0.01$ \\
\hline NGC1350 & $03^{\mathrm{h}} 31^{\mathrm{m}} 08^{\mathrm{s}} .12$ & $-33^{\circ} 37^{\prime} 43^{\prime \prime} .1$ & $10.71 \dagger$ & 602 & $\leq 8.61$ & $\leq-0.38$ \\
\hline ESO359 G3 & $03^{\mathrm{h}} 52^{\mathrm{m}} 00^{\mathrm{s}} .92$ & $-33^{\circ} 28^{\prime} 03^{\prime \prime} .5$ & $10.11_{-0.02}^{+0.01 \ddagger}$ & 130 & $\leq 7.95$ & $\leq 0.05$ \\
\hline FCCB $857 / 858 *$ & $03^{\mathrm{h}} 33^{\mathrm{m}} 19.49$ & $-35^{\circ} 20^{\prime} 41^{\prime \prime} .4$ & $9.31_{-0.04}^{+0.01 \ddagger}$ & 34 & $\leq 7.36$ & $\leq-0.07$ \\
\hline FCCB950 & $03^{\mathrm{h}} 34^{\mathrm{m}} 31^{\mathrm{s}} .65$ & $-36^{\circ} 52^{\prime} 20^{\prime \prime} .7$ & $9.47_{-0.04}^{+0.02 \ddagger}$ & 31 & $\leq 7.32$ & $\leq-0.64$ \\
\hline FCCB990 & $03^{\mathrm{h}} 35^{\mathrm{m}} 11^{\mathrm{s}} .38$ & $-33^{\circ} 22^{\prime} 25^{\prime \prime} 6$ & $9.39_{-0.04}^{+0.02 \ddagger}$ & 97 & $\leq 7.82$ & $\leq 0.04$ \\
\hline FCCB713** & $03^{\mathrm{h}} 31^{\mathrm{m}} 20^{\mathrm{s}} .94$ & $-35^{\circ} 29^{\prime} 29^{\prime \prime} .9$ & - & 55 & $\leq 7.57$ & $\leq-0.59$ \\
\hline FCCB792** & $03^{\mathrm{h}} 32^{\mathrm{m}} 25^{\mathrm{s}} .95$ & $-38^{\circ} 05^{\prime} 33^{\prime \prime} .8$ & - & 21 & $\leq 7.16$ & $\leq-0.48$ \\
\hline FCCB1317** & $03^{\mathrm{h}} 39^{\mathrm{m}} 11^{\mathrm{s}} .70$ & $-33^{\circ} 31^{\prime} 56^{\prime \prime} 0$ & - & 97 & $\leq 7.81$ & $\leq 0.10$ \\
\hline
\end{tabular}

1: Name of the galaxy observed; 2: right ascension; 3: declination; 4: stellar mass (see Section 4.3); 5: rms in the spectrum; 6: derived molecular gas mass (see Section 4.3); 7: $\mathrm{H}_{2}$ deficiency (see Section 4.4). $*$ FCCB857 and FCCB858 are close to each other on the sky and were therefore contained within one beam. The stellar mass quoted here is the addition of the stellar masses of both galaxies. The coordinates of FCCB858 are quoted here. $* *$ These galaxies were observed but later found to be background objects. They are therefore omitted in Figs 8 and 9, and stellar masses were therefore not determined for them. $†$ Stellar masses from Fuller et al. (2014). $\ddagger$ Stellar masses derived from $3.6 \mu \mathrm{m}$ images (see Section 4.3).

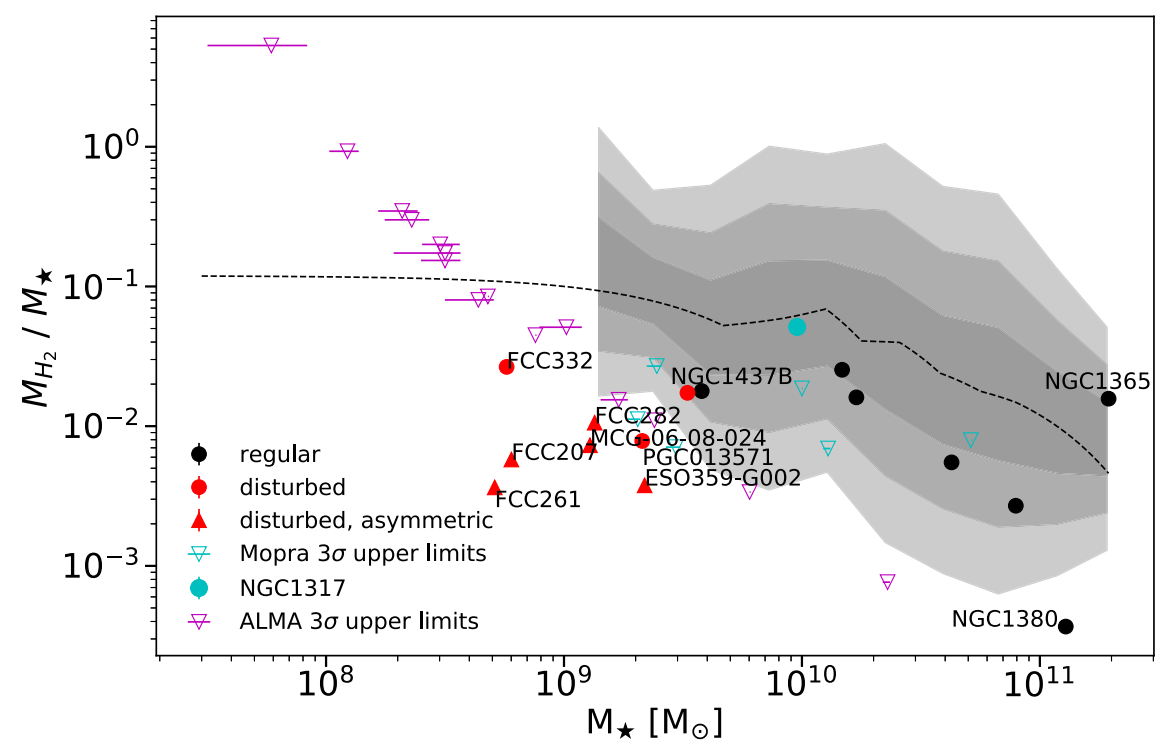

Figure 8. Molecular gas fraction, as a function of stellar mass. Black dots are regular galaxies and red markers are disturbed galaxies. The shape of the marker indicates whether the galaxy may be undergoing ram pressure stripping, based on visual inspection. ALMA upper limits are shown as magenta open triangles and Mopra upper limits as cyan open triangles. The Mopra detection of NGC1317 is shown as a cyan dot. Within the shaded area, the dashed line represents the expected gas fraction based on Saintonge et al. (2017). The three shades of grey indicate the 1,2, and $3 \sigma$ levels (from the inside out) of the xCOLD GASS data. Outside the shaded area the dashed line is based on linear extrapolation (in logspace). Galaxies with high deficiencies and the galaxies that were classified as disturbed are labelled. There is a discrepancy between the expected gas fractions and the gas fractions observed; especially the disturbed galaxies are $\mathrm{H}_{2}$ deficient compared to field galaxies.

H I-line data, obtained with the National Radio Astronomy Observatory (NRAO) Very Large Array. It has high sensitivity $(\leq 1.1 \mathrm{mJy}$ beam $^{-1}$ per channel), high spectral resolution $\left(\leq 2.6 \mathrm{~km} \mathrm{~s}^{-1}\right)$, and high angular resolution $(\sim 6 \mathrm{arcsec})$, resulting in detailed intensity and velocity maps. If the molecular gas in a galaxy is disturbed, we expect its atomic gas to be disturbed as well. Therefore, this comparison, although not ideal, is still meaningful. Categorizing the LITTLE THINGS dwarfs in the same way as the AlFoCS galaxies (see above, Section 5.1), only about half of these dwarf galaxies show disturbed $\mathrm{H}$ I kinematics and morphologies. Since all AlFoCS galaxies with stellar masses lower than $3 \times 10^{9} \mathrm{M}_{\odot}$ have disturbed morphologies and kinematics, this indicates that these low-mass galaxies are more disturbed than their counterparts in the field. This suggests that Fornax is still a very active environment, having significant effects on its members. Furthermore, it implies that less massive galaxies are more susceptible to the effects of the 


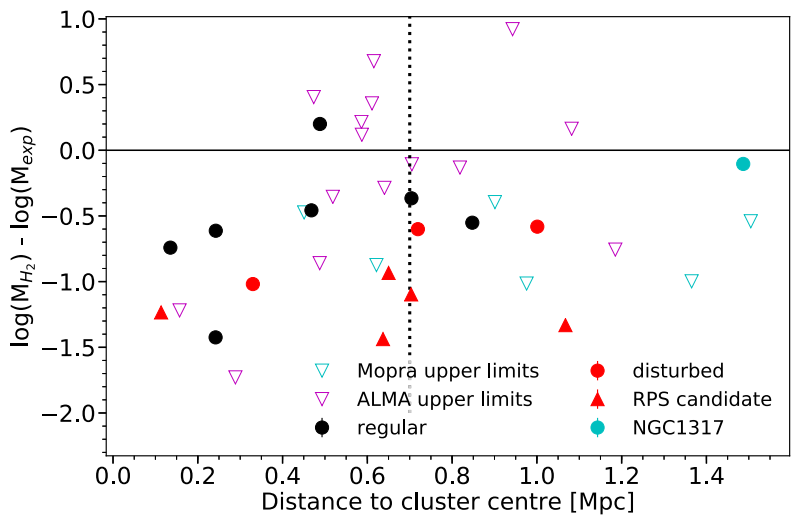

Figure 9. Molecular gas mass deficiencies (see Section 4.4) as a function of the (projected) distance to the cluster centre (defined as the location of NGC1399). Marker shapes and colours are the same as in Fig. 8. There is no clear correlation between a galaxy's $\mathrm{H}_{2}$ deficiency and its distance from the cluster centre.

cluster environment, likely because of their shallower potential wells. This difference in gas deficiency between massive and less massive galaxies is also seen in simulations (e.g. van de Voort et al. 2017), and is likely driven by their shallower potential wells.

\subsection{Ram pressure stripping or galaxy-galaxy interactions?}

The AlFoCS galaxies with disturbed molecular gas reservoirs are $\mathrm{H}_{2}$ deficient compared to field galaxies (see Fig. 8 and Section 4.4). This confirms the result from Horellou et al. (1995), who find that the $\mathrm{CO}$ emission in Fornax cluster galaxies is relatively weak and the $\mathrm{H}_{2}$ masses relatively low. AlFoCS galaxies have deficiencies up to -1.1 dex (see Section 4.4). These deficiencies are higher than those found in Boselli et al. (2014), who find $\mathrm{H}_{2}$ deficiencies of a factor $\sim 2$ for the most H I-deficient galaxies in the Virgo cluster. The molecular gas in the most deficient AlFoCS galaxies is centrally located and asymmetric. Mechanisms that are possibly responsible for this include ram pressure stripping and galaxy-galaxy interactions

Two of the irregular galaxies, MCG-06-08-024 and ESO359-G002, show molecular gas tails that extend well beyond the brightest parts of the galaxy's stellar body (see Section A0.2). Together with the dwarfs FCC207 and FCC261, they have the lowest gas fractions of the disturbed galaxies (see Fig. 8 and Table 3). In both cases, this tail is aligned with the direction of the cluster centre (see Section A0.2). This, in combination with their low gas fractions, can be interpreted as a sign of ongoing ram pressure stripping. This is striking, since RPS is not thought to affect the molecular gas much, as it is bound much more tightly to the galaxy than the atomic gas. Moreover, RPS is thought to be less important in the Fornax cluster than in, for example, the Virgo cluster, given its relatively small size and large density of galaxies (see Section 1).

The fact that the gas tails align with the direction of the cluster centre is, however, not necessarily proof that ram pressure stripping is in play. There are confirmed RPS tails pointing in all directions, even nearly perpendicular to the direction of the cluster centre (e.g. Kenney et al. 2014). This is also seen in simulations (e.g. Yun et al. 2018). Moreover, the kinematics of these galaxies are more irregular than expected based on RPS alone, which suggests that a past galaxy-galaxy interaction may be (co-)responsible for this. In deep FDS images (Iodice et al. 2018), MCG-06-08-024 shows a very disturbed morphology in the outskirts, which could indicate a past galaxy-galaxy interaction. Furthermore, these RPS candidates are not necessarily close to the cluster centre, nor do they have particularly high velocities, as one might expect for galaxies that are undergoing RPS. However, Jaffé et al. (2018), recently found galaxies undergoing RPS all over the cluster, and also in a wide variety of locations in the velocity phase space. Simulations by Yun et al. (2018) show that ram pressure stripped galaxies are more common beyond half the virial radius, where most of the AlFoCS galaxies with disturbed molecular gas are located. Both galaxies discussed here have relatively low masses and shallow potential wells, so they are expected to be susceptible to ram pressure stripping. Yun et al. (2018) also find that galaxies with shallow potential wells can experience extended stripping due to weak ram pressure. Based on these data alone, it is difficult to say whether it is ram pressure affecting these galaxies. The combination with additional data, for example a study of the stellar kinematics of these galaxies, would allow us to distinguish between galaxy-galaxy interactions and ram pressure stripping with more certainty.

Several other galaxies, such as FCC282 and FCC332, also show asymmetric molecular gas reservoirs and were therefore labelled as possible RPS candidates. Asymmetric molecular gas distributions and molecular gas tails can, however, also be the result of galaxy-galaxy interactions. Other galaxies, such as NGC1437B and FCC261, have relatively massive neighbours that are close to them on the sky, which could mean that they are experiencing tidal forces. NGC1437B is the least $\mathrm{H}_{2}$ deficient of the disturbed galaxies (see Fig. 8). If we look at its velocity map and PVD (see Fig. B12), we can see that it has maintained its rotation and still shows a coherent structure, but it appears to be influenced by a pull on its south side. Although a second tail at the north side is missing, this could be an indication of an ongoing tidal interaction. Although the extension of the molecular gas on the south side of the galaxy does not align with the direction of the cluster centre, it is also possible that this asymmetry is caused by RPS, depending on the galaxy's orbit through the cluster (see above). It is currently still relatively far out, located approximately at the virial radius on the sky.

In Fig. 9, there appears to be no correlation between a galaxy's $\mathrm{H}_{2}$ mass deficiency and its distance from the cluster centre. Although we suffer from small number statistics, there are a few other possible explanations for this:

(i) We are looking at a 2D projection of the cluster; the positions of the galaxies along the line of sight are not taken into account.

(ii) Lower mass galaxies end up more $\mathrm{H}_{2}$ deficient than their higher mass counterparts, because of their shallower potential wells. The total $\mathrm{H}_{2}$ mass per galaxy is therefore more a function of their intrinsic mass than of their location in the cluster.

(iii) The responsible mechanism is galaxy-galaxy interactions. While RPS is much more effective in the cluster centre, depending quadratically on the density of the hot halo, galaxy-galaxy interactions are, relatively, more common at the outskirts of the cluster. If the latter play a role, we would expect less of a trend in the gas deficiencies as we moved away from the cluster centre.

(iv) The galaxies are moving through the cluster, so if they experienced RPS when they were near its centre, they could have moved to the outskirts of the cluster since then.

(v) The galaxies were selected to have FIR emission, and therefore the galaxies that lost all their gas are excluded from the sample.

\subsection{Dwarfs}

Among the detections are several galaxies with low stellar masses that can be classified as early-type dwarfs. Four of these have stellar 
masses $M_{\star} \leq 1.0 \times 10^{9} \mathrm{M}_{\odot}$. It was long thought that early-type dwarf galaxies in cluster environments would not have a molecular ISM, due to their expected short stripping time-scales and shallow potentials.

The currently accepted theory is that these galaxies are the remnants of low-mass late-type galaxies that have fallen into the cluster. This hypothesis is supported by the presence of visible structures such as spiral arms, bars, discs, and nuclei and cores (Jerjen, Kalnajs \& Binggeli 2000; Barazza, Binggeli \& Jerjen 2002; De Rijcke et al. 2003; Lisker, Grebel \& Binggeli 2006a; Lisker et al. 2006b), the rotational support of their stellar kinematics (Pedraz et al. 2002; Ryś, Falcón-Barroso \& van de Ven 2013), and the detection of significant amounts of gas and dust in some of them (Conselice 2002; di Serego Alighieri et al. 2007; De Looze et al. 2010, 2013; di Serego Alighieri et al. 2013).

It is also possible that they were already early-type dwarf galaxies to begin with, but reaccreted material through tidal interactions either with the intracluster medium or with another galaxy, which could also trigger new star formation. Inconsistencies in the observations supporting the infalling-spiral scenario (Miller, Lamb \& Cook 1998; Sánchez-Janssen \& Aguerri 2012) may add to the favourability of this idea. However, the limited spatial resolution of most of these studies to date makes it hard to draw strong conclusions.

De Looze et al. (2013) observed 'transition-type dwarf galaxies' (TTDs) in the Virgo cluster. These galaxies are dwarfs that have an apparent early-type morphology but still show dust emission and thus evidence of a cold ISM and star formation. They posit that TTDs are in the process of having their ISM removed by the cluster environment, transforming from late-type dwarfs to quiescent ones (see Boselli et al. 2008 and Koleva et al. 2013 for a more detailed description of the definition and identification of TTDs). They find that many of the dust properties of these objects lie in between what is expected for early-type galaxies and for late-type galaxies, supporting the hypothesis that they are infalling low-mass spirals that are in the process of being quenched. The presence of central cores and dust concentrations is additional evidence in favour of this outside-in gas removal theory.

We suspect that the dwarf galaxies we observe here are TTDs moving through the cluster and being stripped of their gas and thus in the process of being quenched. Each dwarf galaxy observed has a very disturbed and irregular molecular ISM, both morphologically and kinematically, suggesting that it is being stripped by the hot intracluster gas (e.g. MCG-06-08-024 and ESO359-G002; see Figs 4 and B15), or being torn apart by tidal forces. This is in favour of the hypothesis that they are the remnants of infalling gas-rich galaxies. The observation that galaxies with disturbed molecular gas reservoirs seem to favour locations around the virial radius (see Section 4) supports the idea that these dwarfs are starting their first passage through the cluster, or have just crossed it for the first time.

\subsection{NGC1427A}

NGC1427A has been proposed to be undergoing ram pressure stripping (Chanamé, Infante \& Reisenegger 2000; Mora, Chanamé \& Puzia 2015), although recent H I observations by Lee-Waddell et al. (2018) suggest that previous tidal interactions are responsible for the galaxy's irregular shape and for star formation triggering in the disc. It was detected in all five Herschel bands (Fuller et al. 2014). Based on the above observations, the expectation was to detect $\mathrm{CO}$ in this galaxy. However, none was detected.

NGC1427A is vigorously star forming. It is expected to have approximately solar metallicity, based on both its stellar mass and

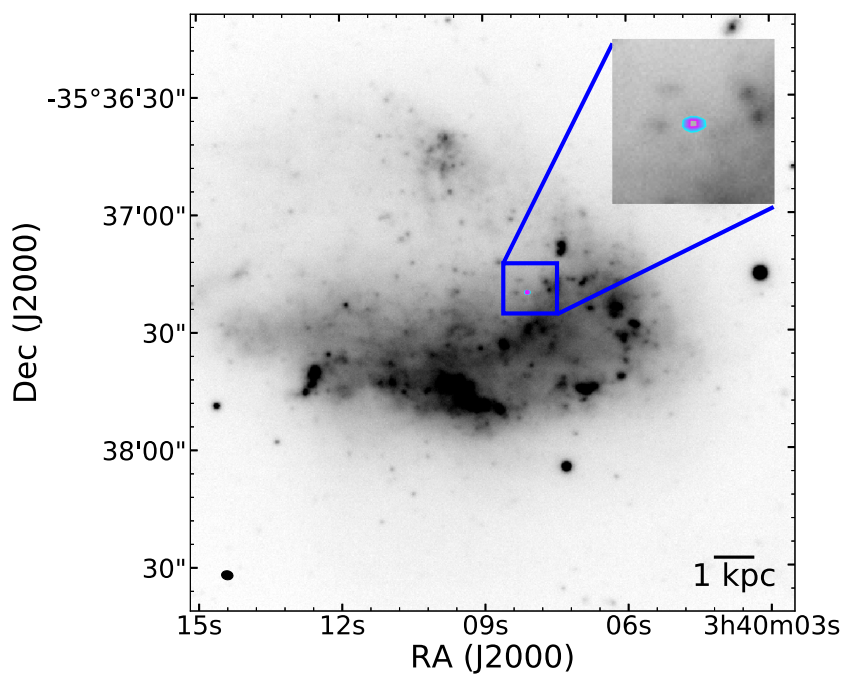

Figure 10. $3 \mathrm{~mm}$ continuum emission in the observation of NGC1427A, overplotted on an optical ( $g$-band) image from the FDS (see Section 3.3). The emission is shown as 10 coloured contours; the lower (outer) contour level equals $4 \sigma$, the inner one $5.6 \sigma$. The emission likely originates from a background source.

analysis of the colours of its star clusters (Mora et al. 2015). Its atomic hydrogen mass is $M_{\mathrm{HI}}=2.1 \times 10^{9} \mathrm{M}_{\odot}$ (Lee-Waddell et al. 2018); we find an upper limit on its molecular hydrogen mass of $M_{\mathrm{H}_{2}}=2.63 \times 10^{7}$, and its SFR is $0.05 \pm 0.03$ (Mora et al. 2015). This leads to the surprisingly high atomic-to-molecular gas ratio $\frac{M_{\mathrm{HI}}}{M_{\mathrm{H}_{2}}}>79$ and short depletion time $\frac{M_{\mathrm{H}_{2}}}{\mathrm{SFR}}<1.3 \mathrm{Gyr}$.

It is possible that the flux is resolved out, since the galaxy extends well beyond the largest recoverable scale of $25 \operatorname{arcsec}$ (see Fig. 10). However, as $\mathrm{CO}$ emission is broken between velocity channels due to its motion within the galaxy, in our mosaic we would still expect to see some emission.

If the $\mathrm{CO}$ emission has not been resolved out, this makes the nondetection of $\mathrm{CO}$ surprising. A natural explanation for this would be if the galaxy underwent a merger or other event that diluted its gas-phase metallicity. We test this by deriving the gas-phase metallicity from the measured gas-to-dust ratio. This is done using the empirical relation between the gas-to-dust ratio and metallicity from Rémy-Ruyer et al. (2014; fig. 4 and table 1) for galaxies in the low-metallicity regime, where we expect the galaxy to be. Using the relation for the higher metallicity regime instead would result in an even lower metallicity, thus only amplifying the analysis below. We use the relation for a metallicity-dependent $X_{\mathrm{CO}}$. Rewriting for the metallicity gives

$12+\log (\mathrm{O} / \mathrm{H})=\frac{-\left(\log \left(\frac{G}{D}\right)-b\right)}{\alpha_{\mathrm{L}}}+x_{\odot}$

where $b=0.96, \alpha_{\mathrm{L}}=3.10 \pm 1.33$, and $x_{\odot}=8.69$ the solar metallicity (Asplund et al. 2009). The gas-to-dust ratio is derived using the $\mathrm{HI}$ and $\mathrm{H}_{2}$ masses stated above and a dust mass of $4.2 \times 10^{6} \mathrm{M}_{\odot}$ from Fuller et al. (2014). Since our upper limit on the $\mathrm{H}_{2}$ mass depends on $X_{\mathrm{CO}}$, we combine the relation above and that between $X_{\mathrm{CO}}$ and metallicity (equation 2) to estimate the metallicity (and thus the resulting $X_{\mathrm{CO}}$ ) in this object. The resulting metallicity, assuming these relations hold, is $12+\log (\mathrm{O} / \mathrm{H})=8.12$ (or $0.27 \mathrm{Z}_{\odot}$ ). This is significantly lower than the metallicity derived from the stellar mass $\left[12+\log (\mathrm{O} / \mathrm{H})=8.71 ; 1.05 \mathrm{Z}_{\odot}\right.$; see Section 4.3] and that found for young star clusters by Mora et al. (2015), who find values 
of $\sim 0.4-1 \mathrm{Z}_{\odot}$. It implies an $X_{\mathrm{CO}}$ of $\sim 18 \times 10^{20} \mathrm{~cm}^{-2}\left(\mathrm{~K} \mathrm{~km} \mathrm{~s}^{-1}\right)^{-1}$ (see Section 4.3), in which case the $\mathrm{H}_{2}$ mass limit we can set in this object increases to $\sim 2.1 \times 10^{8} \mathrm{M}_{\odot}$. These revised values for the atomic-to-molecular gas ratio and the molecular gas depletion time yield $>10$ and $<4.2 \mathrm{Gyr}$, respectively, much more consistent with canonical values.

We do detect a $3 \mathrm{~mm}$ continuum source in our NGC1427A observations. This is shown in Fig. 10, where the continuum is overplotted as coloured contours on a $g$-band image from the FDS, similar to Fig. 7. The emission originates from a source towards the north of NGC1427A, slightly to the east side relative to its centre. There are two possible candidates for its origin: Either the emission comes from a background source or it comes from an AGN associated with NGC1427A that has been moved off-centre as a result of a galaxygalaxy interaction. The latter interpretation seems quite speculative. However, based on the information currently available and the galaxy's turbulent history, it is a possibility. In case the continuum originates from a background source, we would expect to see an optical counterpart in the optical FDS image. These images are quite deep, and can detect point sources down to $\sim 25-26$ mag. However, no optical counterpart is detected. Moreover, no emission lines are detected in archival MUSE observations of this area. Thus, if it is indeed a background object, it must be heavily obscured by dust.

\section{CONCLUSIONS/SUMMARY}

We have presented the first results from the ALMA Fornax Cluster Survey (AlFoCS), a complete survey of the $\mathrm{CO}(1-0)$ in all Fornax cluster galaxies above $>3 \times 10^{8} \mathrm{M}_{\odot}$ that contain dust (Fuller et al. 2014) and/or H I (Waugh et al. 2002; Loni et al. in prep; based on ATCA data). The goal of this survey is to study the effects of the cluster environment on the cold molecular gas inside galaxies. We present moment zero, one, and two maps as well as positionvelocity diagrams, spectra, and comparisons with optical images for all galaxies detected. Furthermore, we estimate $\mathrm{H}_{2}$ masses and derive the corresponding $\mathrm{H}_{2}$ deficiencies compared to field galaxies. The main conclusions from this initial analysis are as follows:

(i) The cold molecular gas in galaxies is indeed affected by the cluster environment. All galaxies with stellar masses below $3 \times 10^{9} \mathrm{M}_{\odot}$ (8 out of 15 detected galaxies) have morphologically and kinematically disturbed gas reservoirs. 'Disturbed' means that their molecular gas is distributed asymmetrically with respect to the optical centre of the galaxy, sometimes with irregular shapes or large tails. The moment one maps and PVDs show irregular motions, and in most cases no rotation can be identified. This suggests that Fornax is still a very active environment, having a significant impact on its members. More massive galaxies are probably experiencing the same interactions; however, the molecular gas may not be affected in the same way, because of their deeper potential wells.

(ii) Continuum was detected in four of the galaxies observed. In three of them it is likely associated with AGN activity. In one case (NGC1427A) the emission does not originate from the centre of the galaxy, but rather from the galaxy's edge. It is unclear whether the source of this emission is a background object or an AGN that was moved off-centre due to a recent galaxy-galaxy interaction.

(iii) Galaxies with regular $\mathrm{CO}$ emission have an average $\mathrm{H}_{2}$ deficiency of -0.50 dex, and galaxies with disturbed $\mathrm{CO}$ emission have an average $\mathrm{H}_{2}$ deficiency of -1.1 dex. AlFoCS galaxies with disturbed molecular gas reservoirs are therefore, with few exceptions, significantly deficient in $\mathrm{H}_{2}$ compared to their counterparts in the field (as probed by the xCOLD GASS sample). (iv) Whether a galaxy has a molecular gas reservoir, and whether that reservoir is disturbed or regular, appears to be independent of the galaxy's location within the cluster. However, the sample size is small.

(v) Two molecular gas tails were detected, which extend beyond the galaxy's stellar body and align with the direction of the cluster centre. These galaxies are possibly undergoing ram pressure stripping. The relatively high $\mathrm{H}_{2}$ deficiencies support this explanation. Several other galaxies have molecular gas reservoirs that are asymmetric with respect to their stellar bodies as well, and are therefore possible RPS candidates; however, it is difficult to draw definite conclusions from these data alone.

To be able to really distinguish between ram pressure stripping and galaxy-galaxy interactions, we need to compare the stellar kinematics (derived from e.g. MUSE observations: Sarzi et al. 2018) of the galaxies in question with the kinematics of their disturbed molecular gas. If both the molecular gas and stars show similar kinematics, ram pressure stripping can be ruled out. If only the molecular gas kinematics are disturbed, on the other hand, we can confirm that ram pressure stripping plays a role. This will be the aim of a future work. Similarly, we will compare our data with those of the Herschel Fornax Cluster Survey (Fuller et al. 2014), to compare the molecular gas and dust distributions, and derive gas-to-dust ratios for the galaxies in our sample.

In conclusion, the detection of a relatively high number of galaxies with disturbed molecular gas reservoirs and $\mathrm{H}_{2}$ deficiencies of sometimes more than an order of magnitude reveals the importance of the cluster environment for even the tightly bound molecular gas phase and motivates further study of environmental effects on the molecular gas in nearby clusters.

\section{ACKNOWLEDGEMENTS}

This publication has received funding from the European Union's Horizon 2020 research and innovation programme under grant agreement No 730562 [RadioNet].

This project has received funding from the European Research Council (ERC) under the European Union's Horizon 2020 research and innovation programme (grant agreement no. 679627; project name FORNAX).

NZ acknowledges support from the European Research Council (ERC) in the form of Consolidator Grant CosmicDust (ERC-2014CoG-647939)

TAD acknowledges support from a Science and Technology Facilities Council Ernest Rutherford Fellowship.

FvdV is supported by the Klaus Tschira Foundation.

R.F.P. acknowledges financial support from the European Union's Horizon 2020 research and innovation programme under the Marie Skłodowska-Curie grant agreement No. 721463 to the SUNDIAL ITN network.

This paper makes use of the following ALMA data: ADS/JAO.ALMA\#2015.1.01135.S. ALMA is a partnership of the ESO (representing its member states), NSF (USA), and NINS (Japan), together with the NRC (Canada), NSC, ASIAA (Taiwan), and KASI (Republic of Korea), in cooperation with the Republic of Chile. The Joint ALMA Observatory is operated by the ESO, AUI/NRAO, and NAOJ.

The Mopra radio telescope is part of the Australia Telescope National Facility, which is funded by the Australian Government for operation as a National Facility managed by CSIRO. 
This publication makes use of data products from the Wide-field Infrared Survey Explorer, which is a joint project of the University of California, Los Angeles, and the Jet Propulsion Laboratory/California Institute of Technology, funded by the National Aeronautics and Space Administration.

This research has made use of the NASA/IPAC Extragalactic Database (NED), which is operated by the Jet Propulsion Laboratory, California Institute of Technology, under contract with the National Aeronautics and Space Administration.

\section{REFERENCES}

Accurso G. et al., 2017, MNRAS, 470, 4750

Alatalo K. et al., 2013, MNRAS, 432, 1796

Asplund M., Grevesse N., Sauval A. J., Scott P., 2009, ARA\&A, 47, 481

Barazza F. D., Binggeli B., Jerjen H., 2002, A\&A, 391, 823

Barnes D. G. et al., 2001, MNRAS, 322, 486

Binggeli B., Sandage A., Tammann G. A., 1985, AJ, 90, 1681

Boizelle B. D., Barth A. J., Darling J., Baker A. J., Buote D. A., Ho L. C., Walsh J. L., 2017, ApJ, 845, 170

Bolatto A. D., Wolfire M., Leroy A. K., 2013, ARA\&A, 51, 207

Booth R. S. et al., 1989, A\&A, 216, 315

Boselli A. et al., 2011, A\&A, 528, A107

Boselli A. et al., 2018, A\&A, 614, A56

Boselli A., Gavazzi G., 2006, PASP, 118, 517

Boselli A., Casoli F., Lequeux J., 1995, A\&AS, 110, 521

Boselli A., Boissier S., Cortese L., Gavazzi G., 2008, A\&A, 489, 1015

Boselli A., Cortese L., Boquien M., Boissier S., Catinella B., Gavazzi G., Lagos C., Saintonge A., 2014, A\&A, 564, A67

Briggs D. S., 1995, AAS Meeting Abstracts, 27, 1444

Brinchmann J., Charlot S., White S. D. M., Tremonti C., Kauffmann G., Heckman T., Brinkmann J., 2004, MNRAS, 351, 1151

Casoli F., Boisse P., Combes F., Dupraz C., 1991, A\&A, 249, 359

Cayatte V., van Gorkom J. H., Balkowski C., Kotanyi C., 1990, AJ, 100, 604

Chanamé J., Infante L., Reisenegger A., 2000, ApJ, 530, 96

Coe D., 2010, preprint (arXiv:1005.0411)

Colless M. et al., 2001, MNRAS, 328, 1039

Conselice C. J., 2002, ApJ, 573, L5

Cortese L. et al., 2010, A\&A, 518, L49

Cortese L. et al., 2012, A\&A, 540, A52

Cortés J. R., Kenney J. D. P., Hardy E., 2006, AJ, 131, 747

Cowie L. L., Songaila A., 1977, Nature, 266, 501

Croom S., Saunders W., Heald R., 2004, Anglo-Australian Observatory Epping Newsletter, 106, 12

Dame T. M., 2011, preprint (arXiv:1101.1499)

Davies J. I. et al., 2010, A\&A, 518, L48

Davies J. I. et al., 2013, MNRAS, 428, 834

De Looze I. et al., 2010, A\&A, 518, L54

De Looze I. et al., 2013, MNRAS, 436, 1057

De Rijcke S., Dejonghe H., Zeilinger W. W., Hau G. K. T., 2003, A\&A, 400, 119

di Serego Alighieri S. et al., 2007, A\&A, 474, 851

di Serego Alighieri S. et al., 2013, A\&A, 552, A8

Dressler A., 1980, ApJ, 236, 351

Drinkwater M. J., Sadler E. M., Davies J. I., Dickens R. J., Gregg M. D., Parker Q. A., Phillipps S., Smith R. M., 1999, in Morganti R., Couch W. J., eds, Looking Deep in the Southern Sky, Proceedings of the ESO/Australia Workshop. Springer-Verlag, Berlin, p. 21

Drinkwater M. J., Gregg M. D., Colless M., 2001, ApJ, 548, L139

Elbaz D. et al., 2007, A\&A, 468, 33

Ferguson H. C., 1989, AJ, 98, 367

Ferrarese L. et al., 2012, ApJS, 200, 4

Fujita Y., 2004, PASJ, 56, 29

Fuller C. et al., 2014, MNRAS, 440, 1571

Fumagalli M., Krumholz M. R., Prochaska J. X., Gavazzi G., Boselli A., 2009, ApJ, 697, 1811
Gavazzi G., Boselli A., van Driel W., O’Neil K., 2005, A\&A, 429, 439

Gunn J. E., Gott J. R., III, 1972, ApJ, 176, 1

Hayashi M. et al., 2018, ApJ, 856, 118

Haynes M. P., Giovanelli R., Chincarini G. L., 1984, ARA\&A, 22, 445

Hinton S. R., Davis T. M., Lidman C., Glazebrook K., Lewis G. F., 2016, Astron. Comput., 15, 61

Högbom J. A., 1974, A\&AS, 15, 417

Horellou C., Casoli F., Dupraz C., 1995, A\&A, 303, 361

Huchra J. P. et al., 2012, ApJS, 199, 26

Hunter D. A. et al., 2012, AJ, 144, 134

Iodice E. et al., 2016, ApJ, 820, 42

Iodice E. et al., 2017, ApJ, 839, 21

Iodice E. et al., 2018, A\&A, preprint (arXiv:1812.01050)

Jaffé Y. L. et al., 2018, MNRAS, 476, 4753

Jerjen H., Kalnajs A., Binggeli B., 2000, A\&A, 358, 845

Jordán A. et al., 2007, ApJS, 171, 101

Kenney J. D. P., Young J. S., 1989, ApJ, 344, 171

Kenney J. D. P., Geha M., Jáchym P., Crowl H. H., Dague W., Chung A., van Gorkom J., Vollmer B., 2014, ApJ, 780, 119

Koleva M., Bouchard A., Prugniel P., De Rijcke S., Vauglin I., 2013, MNRAS, 428, 2949

Kuijken K. et al., 2002, The Messenger, 110, 15

Ladd N., Purcell C., Wong T., Robertson S., 2005, PASA, 22, 62

Larson R. B., Tinsley B. M., Caldwell C. N., 1980, ApJ, 237, 692

Laurikainen E., Salo H., Buta R., Knapen J., Speltincx T., Block D., 2006, AJ, 132, 2634

Lee B. et al., 2017, MNRAS, 466, 1382

Lee-Waddell K. et al., 2018, MNRAS, 474, 1108

Lena D. et al., 2015, ApJ, 806, 84

Lewis I. J. et al., 2002, MNRAS, 333, 279

Lisker T., Grebel E. K., Binggeli B., 2006a, AJ, 132, 497

Lisker T., Glatt K., Westera P., Grebel E. K., 2006b, AJ, 132, 2432

McMullin J. P., Waters B., Schiebel D., Young W., Golap K., 2007, in Shaw R. A., Hill F., Bell D. J., eds, ASP Conf. Ser. Vol. 376, Astronomical Data Analysis Software and Systems XVI. Astron. Soc. Pac., San Francisco, p. 127

Mihos J. C., 2004, Clusters of Galaxies: Probes of Cosmological Structure and Galaxy Evolution. Carnegie Observatories Astrophysics Series, 3, p. 277

Miller M. C., Lamb F. K., Cook G. B., 1998, ApJ, 509, 793

Moore B., Katz N., Lake G., Dressler A., Oemler A., 1996, Nature, 379, 613

Mora M. D., Chanamé J., Puzia T. H., 2015, AJ, 150, 93

Navarro J. F., Frenk C. S., White S. D. M., 1997, ApJ, 490, 493

Noble A. G. et al., 2018, preprint (arXiv:1809.03514)

Noeske K. G. et al., 2007, ApJ, 660, L43

Nulsen P. E. J., 1982, MNRAS, 198, 1007

Oemler A., Jr., 1974, ApJ, 194, 1

Paolillo M., Fabbiano G., Peres G., Kim D.-W., 2002, ApJ, 565, 883

Pedraz S., Gorgas J., Cardiel N., Sánchez-Blázquez P., Guzmán R., 2002, MNRAS, 332, L59

Peng C. Y., Ho L. C., Impey C. D., Rix H.-W., 2002, AJ, 124, 266

Peng C. Y., Ho L. C., Impey C. D., Rix H.-W., 2010, AJ, 139, 2097

Pettini M., Pagel B. E. J., 2004, MNRAS, 348, L59

Pilbratt G. L. et al., 2010, A\&A, 518, L1

Rémy-Ruyer A. et al., 2014, A\&A, 563, A31

Robotham A. S. G. et al., 2011, MNRAS, 416, 2640

Rodríguez-Ardila A., Prieto M. A., Mazzalay X., Fernández-Ontiveros J. A., Luque R., Müller-Sánchez F., 2017, MNRAS, 470, 2845

Rudnick G. et al., 2017, ApJ, 849, 27

Ryś A., Falcón-Barroso J., van de Ven G., 2013, MNRAS, 428, 2980

Saintonge A. et al., 2011, MNRAS, 415, 32

Saintonge A. et al., 2017, ApJS, 233, 22

Sánchez-Janssen R., Aguerri J. A. L., 2012, MNRAS, 424, 2614

Sánchez S. F. et al., 2017, MNRAS, 469, 2121

Sarzi M. et al., 2018, A\&A, 616, A121

Sault R. J., Teuben P. J., Wright M. C. H., 1995, in Shaw R. A., Payne H. E., Hayes J. J. E., eds, ASP Conf. Ser. Vol. 77, Astronomical Data Analysis Software and Systems IV. Astron. Soc. Pac., San Francisco, p. 433 
Saunders W. et al., 2004, in Moorwood A. F. M., Iye M., eds, Proc. SPIE Conf. Ser. Vol. 5492, Ground-based Instrumentation for Astronomy. SPIE, Bellingham, p. 389

Scharf C. A., Zurek D. R., Bureau M., 2005, ApJ, 633, 154

Schindler S., Binggeli B., Böhringer H., 1999, A\&A, 343, 420

Schipani P. et al., 2012, Mem. S. A. It. Suppl., 19, 393

Serra P. et al., 2016, in Proceedings of MeerKAT Science: On the Pathway to the SKA. 25-27 May, 2016 Stellenbosch, South Africa (MeerKAT2016). p. 8. https://pos.sissa.it/cgi-bin/reader/conf.cgi? confid = 277, id.8.

Sharp R. et al., 2006, in McLean I. S., Iye M., eds, Proc. SPIE Conf. Ser. Vol. 6269, Ground-Based and Airborne Instrumentation for Astronomy. SPIE, Bellingham, p. $62690 \mathrm{G}$

Shull J. M., 2014, ApJ, 784, 142

Solanes J. M., Manrique A., García-Gómez C., González-Casado G., Giovanelli R., Haynes M. P., 2001, ApJ, 548, 97

Stach S. M., Swinbank A. M., Smail I., Hilton M., Simpson J. M., Cooke E. A., 2017, ApJ, 849, 154

Stark A. A., Knapp G. R., Bally J., Wilson R. W., Penzias A. A., Rowe H. E., 1986, ApJ, 310, 660

Tonry J. L., Dressler A., Blakeslee J. P., Ajhar E. A., Fletcher A. B., Luppino G. A., Metzger M. R., Moore C. B., 2001, ApJ, 546, 681

van de Voort F., Bahé Y. M., Bower R. G., Correa C. A., Crain R. A., Schaye J., Theuns T., 2017, MNRAS, 466, 3460

Venhola A. et al., 2017, A\&A, 608, A142

Venhola A. et al., 2018, preprint (arXiv:1810.00550)

Vollmer B., Braine J., Pappalardo C., Hily-Blant P., 2008, A\&A, 491, 455

Wang T. et al., 2018, ApJ, 867, L29

Waugh M. et al., 2002, MNRAS, 337, 641

Wright E. L. et al., 2010, AJ, 140, 1868

Young L. M. et al., 2011, MNRAS, 414, 940

Yun K. et al., 2018, preprint (arXiv:1810.00005)

Zabludoff A. I., Mulchaey J. S., 1998, ApJ, 498, L5

\section{SUPPORTING INFORMATION}

Supplementary data are available at MNRAS online.

Figure S1: B1a: three-colour image of ESO358-G063. B1b: moment zero map: distribution of the cold molecular gas as traced by the ALMA CO data. B1c: moment 1 map: velocity map of the cold molecular gas. Each colour represents a $10 \mathrm{~km} \mathrm{~s}^{-1}$ velocity channel. B1d: moment 2 map: line width of the CO integrated spectrum. B1e: Position-velocity diagram of the cold molecular gas. The uncertainties in the spatial and velocity directions are indicated in the upper right corner. B1f: $\mathrm{CO}(1-0)$ line. The beam of the observations is shown in the lower left corners of the moment maps, as well as a $1 \mathrm{kpc}$ scale bar in the lower right corners. The beam of the observations is shown in the lower left corners of the moment maps, as well as a $1 \mathrm{kpc}$ scale bar in the lower right corners.

Figure S2: MGC-06-08-024, similar to Fig. B1.

Figure S3: NGC1365, similar to Fig. B1.

Figure S4: NGC1380, similar to Fig. B1.

Figure S5: NGC1386, similar to Fig. B1.

Figure S6: NGC1387, similar to Fig. B1.

Figure S7: FCC207, similar to Fig. B1, except that the velocity channels are $2 \mathrm{~km} \mathrm{~s}^{-1}$ wide.

Figure S8: FCCFCC261, similar to Fig. B1, except that the velocity channels are $2 \mathrm{~km} \mathrm{~s}^{-1}$ wide.

Figure S9: PGC013571, similar to Fig. B1.

Figure S10: FCC282, similar to Fig. B1.

Figure S11: NGC1436, similar to Fig. B1.

Figure S12: NGC1437B, similar to Fig. B1.

Figure S13: ESO358-G063, similar to Fig. B1.

Figure S14: FCC332, similar to Fig. B1.
Figure S15: ESO359-G002, similar to Fig. B1.

Figure S16: CO integrated intensity contours overplotted on optical ( $g$-band) images of the galaxies (see Section 3.6).

Figure S17: NGC1351A

Figure S18: MCG-06-08-024

Figure S19: NGC1365

Figure S20: NGC1380

Figure S21: NGC1386

Figure S22: NGC1387

Figure S23: FCC207

Figure S24: FCC261

Figure S25: PGC013571

Figure S26: FCC282

Figure S27: NGC1436

Figure S28: NGC1437B

Figure S29: ESO358-G063

Figure S30: FCC 332

Figure S31: ESO359-G002

Please note: Oxford University Press is not responsible for the content or functionality of any supporting materials supplied by the authors. Any queries (other than missing material) should be directed to the corresponding author for the article.

\footnotetext{
${ }^{1}$ School of Physics and Astronomy, Cardiff University, Queen's Building, The Parade, Cardiff CF24 3AA, Wales, UK

${ }^{2} A S T R O N$, the Netherlands Institute for Radio Astronomy, Oude Hoogeveensedijk 4, NL-7991 PD Dwingeloo, The Netherland

${ }^{3}$ Faculty of Physics, Ludwig-Maximilians-Universität, Scheinerstr. 1, D81679 Munich, Germany

${ }^{4}$ UK ALMA Regional Centre Node, Jodrell Bank Centre for Astrophysics, School of Physics and Astronomy, The University of Manchester, Oxford Road, Manchester M13 9PL, UK

${ }^{5}$ Kapteyn Astronomical Institute, University of Groningen, PO Box 72, $\mathrm{NL}$ 9700 AB Groningen, The Netherlands

${ }^{6}$ INAF-Astronomical Observatory of Capodimonte, via Moiariello 16, Naples I-80131, Italy

${ }^{7}$ Astronomy Research Unit, University of Oulu, FI-90014 Oulu, Finland

${ }^{8}$ Sterrenkundig Observatorium, Department of Physics and Astronomy, Universiteit Gent, Krijgslaan 281 S9, B-9000 Gent, Belgium

${ }^{9}$ Department of Physics and Astronomy, University College London, Gower Street, London WC1E 6BT, UK

${ }^{10}$ Sterrenkundig Observatorium, Universiteit Gent, Krijgslaan 281 S9, B9000 Gent, Belgium

${ }^{11}$ Institute of Astronomy, University of Cambridge, Madingley Road, Cambridge CB3 OHA, UK

${ }^{12}$ Observatório do Valongo, Universidade Federal do Rio de Janeiro, Ladeira Pedro Antônio 43, 20080-090 Rio de Janeiro, RJ, Brazil

${ }^{13}$ Astronomy Department, Yale University, P.O. Box 208101, New Haven, CT 06520-8101, USA

${ }^{14}$ INAF - Osservatorio Astronomico di Cagliari, Via della Scienza 5, I-09047

Selargius (CA), Italy

${ }^{15}$ Heidelberg Institute for Theoretical Studies, Schloss-Wolfsbrunnenweg 35, D-69118 Heidelberg, Germany

${ }^{16}$ Astronomy Department, Yale University, PO Box 208101, New Haven, CT 06520-8101, USA

${ }^{17}$ National Radio Astronomy Observatory, 520 Edgemont Road, Charlottesville, VA 22903-2475, USA

${ }^{18}$ Physics Department, New Mexico Tech, 801 Leroy Place, Socorro, NM 87801, USA

${ }^{19}$ National Radio Astronomy Observatory, Socorro, NM 87801, USA
}

This paper has been typeset from a $\mathrm{T}_{\mathrm{E}} \mathrm{X} / \mathrm{LT}_{\mathrm{E}} \mathrm{X}$ file prepared by the author. 